\title{
高性能贵金属/氧化铈纳米催化材料的控制合成、 性能调控及应用的研究进展
}

\author{
张茜, 张亚文 ${ }^{*}$
}

北京分子科学国家研究中心, 北京大学稀土材料化学及应用国家重点实验室, 北京大学化学与分子工程学院, 北京 100871

*通讯作者, E-mail: ywzhang@pku.edu.cn

收稿日期: 2020-07-15; 接受日期: 2020-08-11; 网络版发表日期: 2020-09-15

国家重点基础研究发展规划(编号: 2016YFB0701100)和国家自然科学基金(编号: 21832001, 21771009)资助项目

摘要贵金属由于其独特的物理化学性质而被广泛应用于多个领域，但是其在地壳中储量较低、价格昂贵、 单一组分易于团聚和烧结等缺点限制了其大规模催化应用。通过构建金属合金纳米晶和贵金属负载型催化剂可 以有效解决上述问题。本文系统概述了贵金属/氧化铈纳米催化材料的控制合成和性能调控，结合本课题组的相 关研究工作，主要介绍了特定形貌和纳米结构的双金属/三金属纳米催化剂和贵金属-氧化铈负载型催化剂的控 制合成、结构对性能的影响以及它们在催化领域的应用进展. 最后展望了关于贵金属/氧化铈纳米催化材料的前 沿研究的发展前景。

关键词贵金属, 氧化铈, 控制合成, 催化性能

\section{1 引言}

随着经济和科技的迅速发展，人们对于催化剂的 高效节能、绿色环保和简便易行的可控合成等方面的 要求越来越高. 贵金属 $(\mathrm{Pt} 、 \mathrm{Pd} 、 \mathrm{Au} 、 \mathrm{Ag} 、 \mathrm{Ir} 、 \mathrm{Rh}$ 、 $\mathrm{Ru}$ 等)是一类常见的催化功能材料, 由于其独特的物 理和化学性质而受到科研工作者的重视，并被广泛应 用于催化、燃料电池、生物成像、气体传感等领 域 ${ }^{[1 \sim 5]}$. 通过对贵金属的尺寸和形貌进行调控，可以进 一步提高催化剂的活性和选择性. 然而单一贵金属组 分在催化反应中容易发生团聚、烧结和毒化等使催化 剂失活，同时，贵金属在地壳中储量较低、价格昂贵，
限制了其大规模推广使用. 因此, 如何在保证催化剂的 催化性能满足使用要求的同时降低贵金属的用量成为 亟待解决的重要课题.

通过往一种价格昂贵的贵金属组分中引入另一种 或两种价格比较便宜的贵金属或贱金属形成双金属/ 三金属纳米催化剂是一种可以显著改善贵金属催化性 能并降低其用量的有效方法 ${ }^{[6]}$, 目前已有大量的相关 研究工作被报道. 例如, 向 $\mathrm{Pt}$ 中引入过渡金属 $(\mathrm{Cu}$ 、 Co、Ni等)形成双金属合金, 可以通过微应变、配体 构型、配位数或表面无序等效应来提高Pt原子的利用 率，从而极大地增强其对氧气还原反应(ORR)的电催 化活性 ${ }^{[7]}$; 以 $\mathrm{Pt}$ 或Pd作为活性金属, 引入第二金属作为

引用格式: Zhang Q, Zhang Y. Research progress on high-performance noble metal/cerium oxide nanocatalysts: shape controlled synthesis, performance regulation and applications. Sci Sin Chim, 2020, 50: 1600-1618, doi: 10.1360/SSC-2020-0135 
助剂(如Pd-Zn、Pt-Ga、Pt-Cu、Pd-In等)可以使得轻 烷烃脱氢反应初始催化选择性达到 $90 \%$ 以上 ${ }^{[8]}$; $\mathrm{Pt}$ 和 $\mathrm{Sn}$ 的合金化可以提高催化剂的 $\mathrm{C}=\mathrm{O}$ 键加氢能力 ${ }^{[9]}$ 和抗 $\mathrm{CO}$ 中毒能力, 加入第三种金属组分(如Rh、Ir等)有助 于使 $\mathrm{C}-\mathrm{C}$ 键断裂，从而提高乙醇氧化反应(EOR)的电 催化性能 ${ }^{[10,11]}$ 等. 双金属/三金属催化剂的优异催化特 性主要归因于其具有两种或三种金属组分的共同能 带, 通过改变合金的组成、尺寸和形貌能够连续地改 变双金属/三金属纳米晶表面的电子结构和几何构型, 其中杂原子相互作用可以诱导几何效应和/或电子效 应, 发挥金属组分的协同作用和多功能效应, 以改变催 化剂表面的吸附性能和表面反应，进而影响催化剂的 活性、选择性和稳定性等 ${ }^{[12 ~ 14]}$.

为了最大限度地提高贵金属的催化性能和减少其 用量, 将活性中心负载在基底材料上是另一种强烈有 效的手段. 将贵金属 $(\mathrm{Pt} 、 \mathrm{Pd} 、 \mathrm{Au} 、 \mathrm{Ag} 、 \mathrm{Ir} 、 \mathrm{Rh} 、 \mathrm{Ru}$ 等)与金属氧化物 $\left(\mathrm{CeO}_{2} 、 \mathrm{TiO}_{2} 、 \mathrm{SiO}_{2} 、 \mathrm{WO}_{3}\right.$ 等 $)$ 复合, 基于强金属-载体相互作用(SMSI), 能有效提高催化剂 的催化活性、选择性等催化性能 ${ }^{[15 \sim 18]}$. 其中, $\mathrm{CeO}_{2}$ 是 $\mathrm{Ce}$ 的常见化合物, 由于 $\mathrm{Ce}$ 在地壳中储量丰富, 价格低 廉, 具有独特的核外电子构型 $[\mathrm{Xe}] 4 \mathrm{f}^{1} 5 \mathrm{~d}^{1} 6 \mathrm{~s}^{2}$, 以及 $\mathrm{Ce}^{3+}$ 和 $\mathrm{Ce}^{4+}$ 两种价态, 从而使得 $\mathrm{CeO}_{2}$ 具有独特的物理化学 性质和氧化还原特性, 而被广泛应用于催化领域 ${ }^{[19]}$. 并且, $\mathrm{CeO}_{2}$ 的生长过程易于控制, 可以保持其尺寸和 形貌均一. 将 $\mathrm{CeO}_{2}$ 与贵金属复合得到的负载型催化剂, 在SMSI的作用下, 能够发挥两者的协同作用, 提高催 化性能. 此外, 尺寸效应是影响负载型催化剂催化活性 的重要因素, 贵金属的尺寸会显著影响贵金属纳米粒 子与氧化铈载体之间的SMSI效应, 当其尺寸降至亚纳 米甚至是单原子级别, 会使催化剂表面贵金属物种的 分散度显著增大, 并暴露出更多配位不饱和的原子, 它们会作为反应活性位点使得催化性能发生巨大转 变 ${ }^{[20]}$. 因此，优化贵金属的粒径是提高其催化性能的 有效途径. 相应的, 实现不同尺寸催化剂的可控合成 也是目前面临的难点之一.

本文从贵金属基纳米材料出发, 结合本课题组的 相关研究工作, 主要介绍了贵金属/氧化铈纳米催化剂 (包括双金属/三金属纳米催化剂和贵金属-氧化铈负载 型催化剂)的控制合成、性能调控以及在催化领域的 应用进展, 最后对贵金属/氧化铈纳米催化材料发展的 挑战和前景进行了分析和展望.

\section{2 贵金属/氧化铈纳米催化材料的控制合成}

具有不同表面结构的催化剂在特定的催化反应中 会表现出不同的性能, 而且对于每一个催化反应, 都存 在具有特定组成、结构和尺寸的最佳催化剂. 因此, 选 择和开发合适的合成方法来获得特定形貌的纳米晶体 对于提高催化剂的活性和选择性、理清结构与性能的 关系、制备出最佳结构的催化剂至关重要. 如前文所 述，构建双金属/三金属纳米催化剂和贵金属-氧化铈 负载型催化剂是提高催化剂性能、减少贵金属使用量 和降低催化剂成本的两种重要途径, 下面我们将主要 介绍实现这两种类型催化剂控制合成的常见方法.

\section{1 形貌可控的双金属/三金属纳米催化剂的合成}

对于双金属/三金属纳米晶, 不同的尺寸和形貌使 其具有不同的表面原子排布和电子结构, 因此双金属/ 三金属纳米催化剂的尺寸和形貌对其催化性能具有显 著的影响. 双金属/三金属纳米晶尺寸和形貌的可控合 成, 使其具有多样性的材料结构和种类, 从而可被应用 到不同类型的催化反应中 ${ }^{[6]}$.

制备双金属/三金属纳米晶的方法主要分为物理 方法和化学方法. 在物理合成方法中, 利用机械压力、 高能辐射、热能等使材料粉碎、熔化、蒸发或冷凝产 生纳米晶. 这些方法主要采用由上而下的方法, 没有溶 剂污染, 适用于大规模合成细小的纳米颗粒. 在化学合 成方法中, 主要依靠化学还原剂对金属离子进行还原, 使其成核、生长和稳定. 化学合成法包括化学还原 法、电化学合成法、超声化学合成法、热分解法 等 $^{[21]}$. 其中, 化学还原法是一种应用广泛、适用于双 金属/三金属纳米晶控制合成的有效方法. 在本节中, 我们结合本课题组的工作, 介绍了化学还原法制备不 同尺寸和形貌可控的双金属/三金属纳米结构.

化学还原法具有操作简单、重复性强的特点, 是 制备双金属/三金属纳米晶最常用的方法之一. 在化学 还原方法中, 金属前驱体提供金属离子, 这些离子被还 原剂还原为金属原子，从而使反应溶液混合物中的金 属原子浓度稳定增加. 金属原子浓度的增加导致过饱 和, 随后成核和生长, 其中成核速率和生长速率会显 著影响双金属/三金属纳米晶的尺寸和形貌 ${ }^{[22]}$. 通过改 变反应条件, 如稳定剂和还原剂的用量和种类、金属 离子的浓度、反应温度、时间和 $\mathrm{pH}$ 等, 可以精细地控 
制合金的尺寸、组成和形貌. 在这里可以采用共还原 法或连续还原法, 也可与其他方法结合使用, 来合成 不同尺寸、形貌和结构的双金属/三金属纳米晶. 一般 情况下, 共还原法倾向于得到合金结构纳米晶, 而连续 还原法倾向于得到核壳结构(或表面某种金属组分富 集)的双金属/三金属纳米晶 ${ }^{[23]}$, 如图1所示.

\subsection{1 共还原法}

共还原方法是利用一锅反应来还原存在于同一反 应混合物溶液中的金属盐前驱体. 这种方法合成过程 简单，通过改变稳定剂和还原剂的用量和种类、金属 离子的浓度、反应温度、时间和 $\mathrm{pH}$ 值等反应条件，可 以合成不同组成和形貌的合金纳米晶. 但其还原过程 受还原剂的影响较大：当使用强还原剂时，多金属离 子的还原过程比较迅速, 这样能够有效避免金属原子 单独成核生长，易于形成合金结构. 例如，Zhang等 ${ }^{[24]}$ 以 $\mathrm{NaBH}_{4}$ 为还原剂合成了 Au-Pt-Pd三金属合金，但在 加温下较难对反应过程进行控制，使用温和的还原剂 可以有效控制成核过程.

本课题组 ${ }^{[25]}$ 以聚乙烯吡咯烷酮(PVP)作为还原剂 和包覆剂, 卤化物阴离子 $\left(\mathrm{Br}^{-} / \mathrm{I}^{-}\right)$作为形貌控制剂, 通 过简单的一步水热法合成了具有中空纳米立方体 $(\mathrm{NCs})$ 、纳米二十面体(NIs)和纳米截角八面体(NTOs) 结构的Pd-Rh纳米晶体, 如图2所示. 由于 $\mathrm{Pd}(\mathrm{II}) / \mathrm{Pd}$ 的电 极电位高于 $\mathrm{Rh}(\mathrm{III}) / \mathrm{Rh}, \mathrm{Br}^{-}$离子可以发生(100)面上的 选择性吸附，从而形成Pd-Rh NCs. 在没有KI的情况 下，则会得到Pd-Rh NIs，这表明孪晶种子的去除是由 卤化物离子/氧对的蚀刻效应造成的 ${ }^{[26]}$. 当加入更多的 $\mathrm{KBr}$ 时，由于 $\mathrm{Br}$ 与 $\mathrm{Pd}(\mathrm{II})$ 和 $\mathrm{Rh}(\mathrm{III})$ 协同形成更稳定的配 合物，从而降低了金属离子的还原速率，因此得到了 Pd-Rh NTOs. 所合成的催化剂在Suzuki交叉偶联反应 中表现出不同的催化性能. 而只暴露 $\{100\}$ 晶面的纳米 结构比其他结构表现出更好的催化活性. 通过调控金 属盐前驱体的种类和投入量可以调控合金纳米晶的组 分. 例如, 本课题组 ${ }^{[27]}$ 同样地, 以 PVP作为还原剂和包 覆剂，卤化物阴离子 $\left(\mathrm{Br}^{-} / \mathrm{I}^{-}\right)$作为形貌控制剂，采用一 步水热合成法，通过调控金属盐前驱体的种类，即可 得到组分可变的三金属Pt-Pd-Rh纳米立方体 $(\mathrm{NCs})$ 和 纳米截角八面体(NTOs). 基于实验结果和密度泛函理 论(DFT)模拟，研究了三金属纳米催化剂用于乙醇氧 化反应(EOR)性能的形貌和成分优化. 研究结果表明,

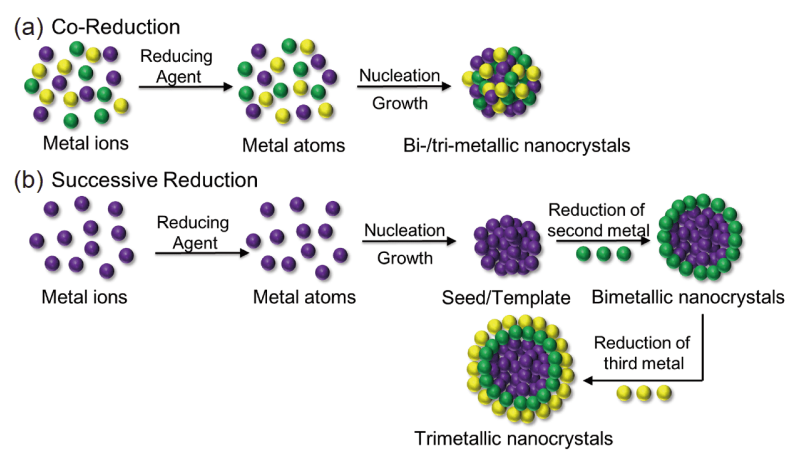

图 1 共还原法( $a)$ 和连续还原法(b)制备双金属/三金属纳米 晶的示意图(网络版彩图)

Figure 1 Schematic illustration of co-reduction (a) and successive reduction (b) methods for the synthesis of bimetallic/trimetallic nanocrystals (color online).

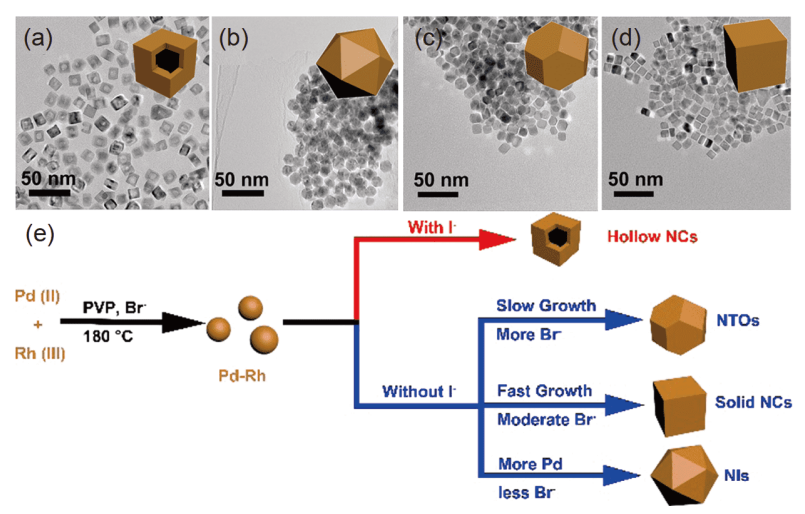

图 2 (a d) 不同形貌Pd-Rh纳米晶的透射电子显微镜( TEM) 图(内插图为几何模型)和(e) 合成示意图 ${ }^{[25]}$ (网络版彩图)

Figure 2 (a d) TEM images (inset shows the geometric model) and (e) scheme of the synthetic procedure for Pd-Rh NPs with various morphologies [25] (color online).

提高EOR中的 $\mathrm{C}-\mathrm{C}$ 键断裂能力的关键因素是纳米晶的 表面成分组成和暴露晶面. 再如, 本课题组 ${ }^{[28]}$ 以 $N, N-$ 二 甲基甲酰胺(DMF)为形貌与结构调控剂，通过一步溶 剂热法合成了一系列几何结构和晶粒尺寸相近的 $\mathrm{Pt}_{3} \mathrm{RhM}$ 纳米合金 $(\mathrm{M}=\mathrm{Fe}, \mathrm{Co}, \mathrm{Ni}, \mathrm{Cu} ; \mathrm{Ga}, \mathrm{In}, \mathrm{Sn}, \mathrm{Pb})$, 如 图3所示. 在低电位条件下, 主族金属在提高EOR的活 性和 $\mathrm{CO}_{2}$ 选择性方面远优于过渡金属.

稳定剂也是影响双金属/三金属纳米晶形貌的重 要因素. 它可以通过选择性稳定配体对合金中的特定 金属的吸引力，选择性地将某一金属拉出到壳中，从 而调控双金属/三金属纳米晶的结构. 例如, 本课题 组 ${ }^{[29]}$ 以 $\mathrm{PVP}$ 为包覆剂, 甲酸铵 $\left(\mathrm{HCOONH}_{4}\right)$ 为还原剂, 引入氨和卤化物离子 $\left(\mathrm{Br}^{-}\right.$或 $\left.\mathrm{Cl}^{-}\right)$与 $\mathrm{Pt}^{2+}$ 配合, 缩小 $\mathrm{Pt}^{2+}$ 


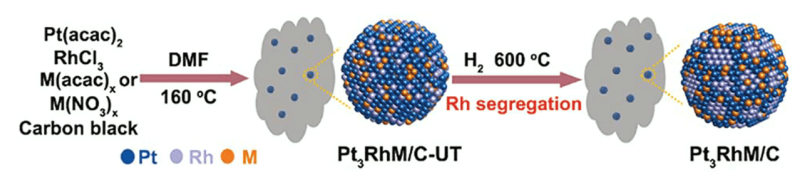

图 $3 \mathrm{Pt}_{3} \mathrm{RhM} / \mathrm{C}$ 三金属纳米催化剂的合成示意图 ${ }^{[28]}$ (网络 版彩图)

Figure 3 Scheme of the synthesis procedure for $\mathrm{Pt}_{3} \mathrm{RhM} / \mathrm{C}$ catalysts [28] (color online).

与 $\mathrm{M}^{2+}$ 前驱体之间的还原电势差即可得到表面富集 $\mathrm{Pt}$ 壳层的Pt-Ni、Pt-Co和Pt-Fe合金纳米晶.

除了上述所提到的结构外, 用共还原法可以合成 已被报道的绝大部分的合金结构. 本课题组 ${ }^{[30]}$ 在油胺 中还原金属前驱体得到了笼状多孔Pt-Cu和树枝状多 孔Pt-Zn、Pt-Ni纳米颗粒, 并通过一步溶剂热法合成了 具有纳米空心球 $(\mathrm{NHSs})$ 、纳米线 $(\mathrm{NWs})$ 和纳米四面体 (NTs) 结构的Ir-Pd合金 ${ }^{[31]}$. 将溶剂热法与化学刻蚀法相 结合, 得到 $\mathrm{Rh}-\mathrm{Cu} 、 \mathrm{Rh}-\mathrm{Ni} 、 \mathrm{Rh}-\mathrm{Pd}-\mathrm{Cu}$ 八面体纳米框 架 ${ }^{[32]}$ 和PtRuCu菱形十二面体纳米框架 ${ }^{[33]}$ 结构. 此外, 我们还报道了一种用于制备形貌可调的单分散Pt-Ir合 金纳米晶的系统合成策略 ${ }^{[34]}$. 如图4所示, 所有的单晶 (纳米八面体、纳米截角八面体和纳米立方体)和多晶 (纳米花、纳米线、纳米短链和纳米八面体星)Pt-Ir纳 米晶均可以通过改变溶剂热过程中的卤化物阴离子 $\left(\mathrm{Br}^{-} / \mathrm{I}^{-}\right)$的浓度来合成. 这些Pt-Ir合金催化剂相比于商 业 $\mathrm{Ir} / \mathrm{C}$ 催化剂表现出更优异的氧析出反应(OER)活性. 特别地, Pt-Ir纳米短链的OER催化活性比商用 $\mathrm{Pt} / \mathrm{C}$ 催 化剂还要高, 这主要是由于其暴露出更高比例的(110) 晶面. 由以上研究工作可以看出, 共还原法是实现金属 合金催化剂形貌可控合成的一种有效方法.

\subsection{2 连续还原法}

连续还原法是利用一种金属的预先形成的纳米粒 子作为优选的种子位点, 并在种子表面沉积其他金属原 子以生长成特定的纳米结构. 这种方法通常用于制备传 统合成方法较难得到的具有特定纳米结构的双金属纳 米晶体. 一般来说, 连续还原法更适用于合成核壳结构 或多级结构的纳米晶 ${ }^{[35]}$. 例如, Schwartz和Michel ${ }^{[36]}$ 通 过连续还原法得到不同结构的 $\mathrm{Au}-\mathrm{Pd}$ 双金属纳米晶, 包括Au核-Pd壳、Pd核-Au壳和合金化纳米颗粒.

连续还原法将纳米晶的成核和生长分离成两个独 立的过程. Jana等 ${ }^{[37]}$ 提出了在 $\mathrm{Au}$ 前驱体盐化学还原成

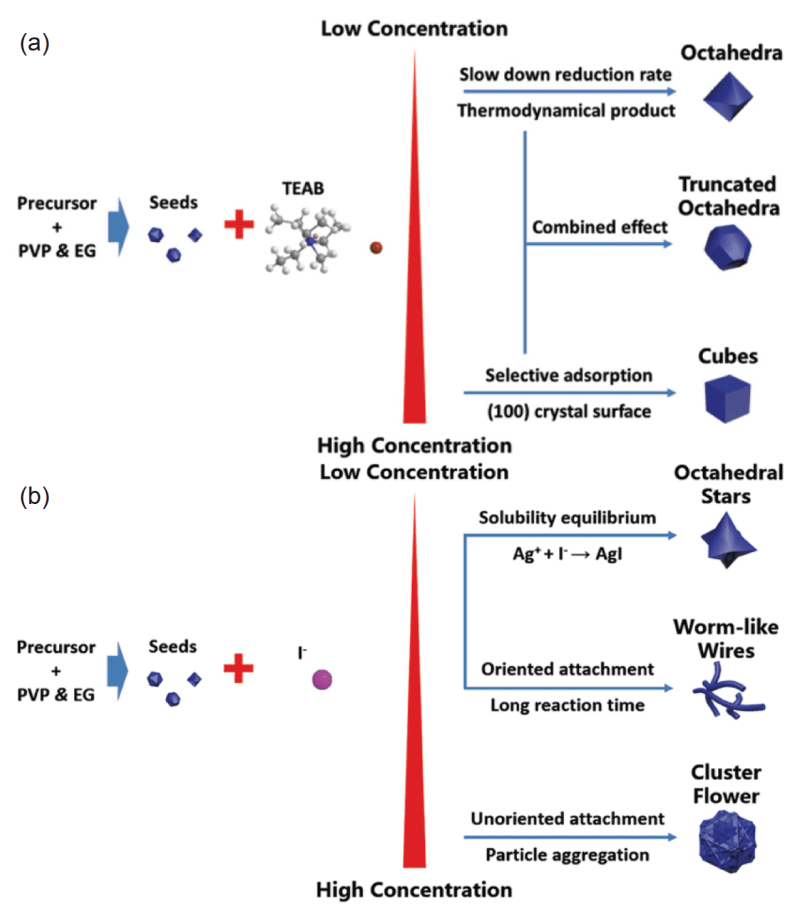

图 4 (a) 单晶Pt-Ir合金纳米晶和(b) 多晶Pt-Ir合金纳米晶的 合成路线图 ${ }^{[34]}$ (网络版彩图)

Figure 4 Scheme of the synthesis procedure for (a) single-crystalline Pt-Ir alloy nanocrystals and (b) polycrystalline Pt-Ir alloy nanocrystals [34] (color online).

$\mathrm{Au}$ 纳米颗粒过程中的种子-介导(seed-mediated)成核的 两步机制: 反应开始时被还原的 $\mathrm{Au}$ 物种缓慢成核，随 后是由种子介导的快速成核和生长. 随着反应的进行, 成核过程经历了一个由快到慢的过程, 最终被生长过 程所取代. Yang 等 ${ }^{[38]}$ 通过这种方法得到了形貌均一的 $\mathrm{Ag} @ \mathrm{Au}$ 核壳纳米线. 研究结果表明, 在碱性条件下, $\mathrm{Au}$ 壳层可以在 $\mathrm{Ag}$ 纳米线上实现保形沉积.

还原剂的选择对于双金属/三金属纳米晶的形貌 控制具有重要影响. 如图5所示, 本课题组 ${ }^{[39]}$ 以甲醛 $(\mathrm{HCHO})$ 为还原剂, 通过 $\mathrm{Na}_{2} \mathrm{C}_{2} \mathrm{O}_{4}$ 配位剂存在下的seedmediated成核过程合成了 $\mathrm{Pt} @ \mathrm{Ru}$ 核壳纳米晶. 在反应 体系中, Pt前驱体先被 100\%还原并作为种子相，此时 $\mathrm{Ru}$ 几乎未被还原. 主要是由于 $\mathrm{C}_{2} \mathrm{O}_{4}{ }^{2-}$ 离子可以与 $\mathrm{Ru}^{n+}$ $(n=2,3)$ 离子形成稳定的配合物以致 $\mathrm{Ru}$ 的还原速率被 减慢, 因此, Pt和 Ru的成核生长时间是分开的. 然后, 面心立方(fcc)结构的Pt种子相可以诱导Ru在其表面外 延生长形成 $\mathrm{Ru}$ 壳层, 得到 $\mathrm{fcc}$ 结构的 $\mathrm{Pt} @ \mathrm{Ru}$ 纳米四 面体.

Galvanic置换法也是连续还原法的一种. 它是由 

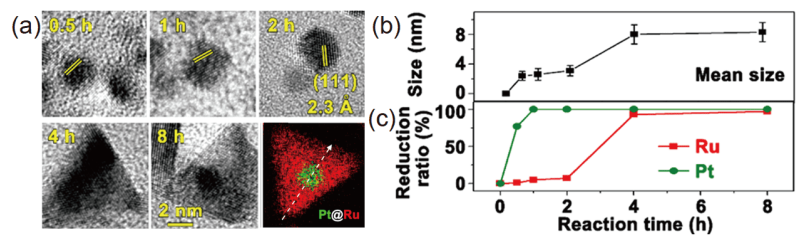

(d)

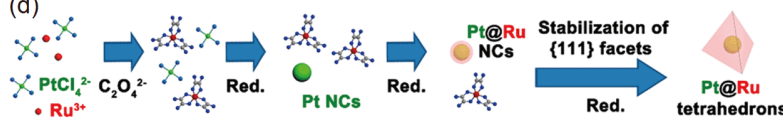

图 $5 \mathrm{Pt}_{10} @ \mathrm{Ru}_{90}$ 纳米四面体在不同反应时间下的 $\mathrm{TEM}$ 图像 (a), 平均粒径(b), 和Pt和 $\mathrm{Ru}$ 的还原效率 (c); (d) Pt@Ru纳米晶 的合成机理图 ${ }^{[39]}$ (网络版彩图)

Figure 5 (a) TEM images, (b) mean size, (c) reduction efficiency of $\mathrm{Pt}$ and $\mathrm{Ru}$ of $\mathrm{Pt}_{10} @ \mathrm{Ru}_{90}$ nanotetrahedrons obtained at different reaction times; (d) schematic illustration of the synthesis mechanism for Pt@Ru nanocrystals [39] (color online).

两种或三种金属之间的氧化还原过程组成，在适当的 驱动力下，氧化还原电位较高的贵金属阳离子有被还 原的趋势. Galvanic置换法是将一种易被氧化的金属 作为牺牲模板和还原剂，同时，另一种还原电位较高 的金属涂敷在模板上被还原，从而合成形貌较为复杂 的双金属/三金属纳米结构(内凹、空心或多孔结构 等). 本课题组 ${ }^{[40]}$ 通过 Galvanic 置换和 $\mathrm{Cu}$ 与 $\mathrm{Au}$ 之间的 Kirkendall效应，合成了孔径为50 100 nm、壁厚为 $4.95 \pm 0.81 \mathrm{~nm}$ 的空心 $\mathrm{Au}-\mathrm{Cu}$ 纳米颗粒. 在制备过程中, 将 $\mathrm{Au}$ 前体溶解在三- $n$-辛基膦(TOP)中以降低其还原电 位, 然后注入铜种子溶液中, 从而使其被 $\mathrm{Cu}^{0}$ 还原. 该空 心 $\mathrm{Au}-\mathrm{Cu}$ 纳米颗粒表现出优异的 $\mathrm{CO}_{2}$ 电还原性能，在 $-0.7 \mathrm{~V}$ ( vs. RHE)的最大CO法拉第效率为 $53.3 \%$. 通过 调控金属盐前驱体的组分和比例、选择不同几何形貌 的牺牲模板可以得到不同组成、形貌和结构的贵金属 合金. 例如，本课题组 ${ }^{[41]}$ 通过对水热合成中反应动力 学和Galvanic置换过程的精细控制，获得了组分可变 的Pt-Cu双金属凹纳米立方体 (CNCs) 和Pt-Pd-Cu三金 属凹纳米立方体. 凹纳米立方体被数个 $\{\mathrm{hk} 0\}$ 高指数台 阶面所包围. X射线光电子能谱(XPS)测试结果表明, 这些纳米晶表面均具有富 $\mathrm{Pt}$ 层，这主要是由于 $\mathrm{Pt}$ 和 $\mathrm{Cu}$ 之间的Galvanic置换过程导致的双金属/三金属纳米晶 表面 $\mathrm{Cu}$ 的去合金化.

总地来说，共还原法和连续还原法都是合成形貌 可控的双金属/三金属纳米晶的常用方法. 双金属纳米 晶的形貌受到诸多参数的影响，例如，金属前驱体(种 类、浓度、还原电位等)、溶剂、还原剂(种类、用 量)、模板/包覆剂、晶种结构、反应温度、反应速
率、时间和 $\mathrm{pH}$ 等. 我们要深刻认识和理解这些参数的 作用机制和影响，从而能够更精确、简便地合成所需 要形貌和结构的催化剂，降低材料合成成本，提高催 化剂的催化性能

\section{2 贵金属-氧化铈负载型催化剂的合成方法}

氧化铈具有优异的由 $\mathrm{Ce}^{3+}$ 和 $\mathrm{Ce}^{4+}$ 可逆转换产生的 储放氧性能，以及可调控的形貌、独特的晶体结构、 丰富的缺陷态和良好的水热稳定性，因此成为一种常 用的催化剂载体．将贵金属负载在氧化铈载体上，既 可以减少贵金属用量、降低催化剂成本，也可以提升 其催化性能. 目前，研究人员已经成功制备了不同形 貌和结构的贵金属-氧化铈复合材料, 其中最常用的方 法有浸渍法、共沉淀法、模板法等.

\subsection{1 浸渍法}

浸渍法是合成贵金属-氧化铈负载型催化剂的一 种很常见的方法，其制备过程简单、反应条件易于控 制. 它是将氧化铈载体(尺寸、形貌和结构已知)放进 贵金属前驱体盐溶液中浸渍，贵金属物种通过物理或 化学作用吸附在载体表面. 通过控制贵金属前驱体的 浓度、用量、浸渍时间和后处理工序等可以实现对贵 金属颗粒尺寸和负载量的调控.

本课题组 ${ }^{[42]}$ 通过浸渍法在 $\mathrm{CeO}_{2}$ 纳米线上负载了 $\mathrm{Ru}$ 单原子 $(\mathrm{SA})$ 和 $\mathrm{Ru}$ 团簇 $(\mathrm{NC})$, 如图6 $(\mathrm{a}, \mathrm{b})$ 所示. $\mathrm{CeO}_{2}$ 纳米线结晶良好，长度约为 $13 \mu \mathrm{m}$, 宽度为 $10 \sim 80 \mathrm{~nm}$. 扩展X射线吸收精细结构结果表明 $\mathrm{Ru}(\mathrm{SA}) / \mathrm{CeO}_{2}$ 样品 中只存在Ru-O配位, 不存在Ru-Ru配位; $\mathrm{Ru}(\mathrm{NC}) / \mathrm{CeO}_{2}$ 中存在少量 $\mathrm{Ru}-\mathrm{Ru}$ 配位. 进一步证实 $\mathrm{Ru}(\mathrm{SA}) / \mathrm{CeO}_{2}$ 中 $\mathrm{Ru}$ 是以单原子形式存在， $\mathrm{Ru}(\mathrm{NC}) / \mathrm{CeO}_{2}$ 中的 $\mathrm{Ru}$ 是团簇结 构, 且团簇大小为 $1.18 \pm 0.25 \mathrm{~nm} . \mathrm{Ru}(\mathrm{SA}) / \mathrm{CeO}_{2}$ 和 $\mathrm{Ru}(\mathrm{NC}) / \mathrm{CeO}_{2}$ 均表现出优异的低温 $\mathrm{CO}_{2}$ 甲烷化活性和 选择性，通过改变 $\mathrm{Ru} / \mathrm{CeO}_{2}$ 中 $\mathrm{Ru}$ 的尺寸，揭示了 SMSI 和 $\mathrm{H}$-溢流效应在 $\mathrm{CO}_{2}$ 甲烷化反应中的竞争关系. Wang 等 ${ }^{[43]}$ 通过浸渍法制备了 $\mathrm{Pt} / \mathrm{CeO}_{2}$ 纳米棒催化剂, $\mathrm{Pt}$ 纳米 颗粒的尺寸约为 $3.34 \mathrm{~nm}$, 分散度为 $33.66 \%, \mathrm{CeO}_{2}$ 纳米 棒的长度为 $120 \pm 50 \mathrm{~nm}$, 宽度为 $13 \pm 5 \mathrm{~nm}$. Shanmugam 等 ${ }^{[44]}$ 先通过水热法制备 $\mathrm{CeO}_{2}$ 纳米棒和纳米立方体载 体，然后通过浸渍法在其表面负载了高度分散且尺寸 可控的 $\mathrm{Rh}$ 金属纳米颗粒. 如图6(c,d)所示, $\mathrm{CeO}_{2}$ 纳米 立方体的平均尺寸为 $33 \mathrm{~nm}, \mathrm{CeO}_{2}$ 纳米棒的平均直径 


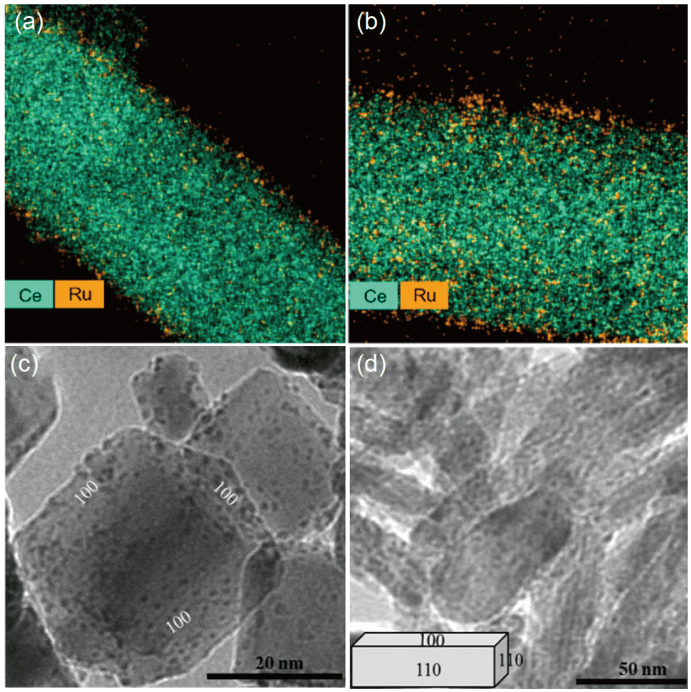

图 6 (a) $\mathrm{Ru}(\mathrm{SA}) / \mathrm{CeO}_{2}$, (b) $\mathrm{Ru}(\mathrm{NC}) / \mathrm{CeO}_{2}$ 的元素分布图 ${ }^{[42]}$; (c) $\mathrm{Rh} / \mathrm{CeO}_{2}-\mathrm{NC}$, (d) $\mathrm{Rh} / \mathrm{CeO}_{2}-\mathrm{NR}$ 的TEM图像 ${ }^{[4]}$ (网络版彩 图)

Figure 6 The element maps of (a) $\mathrm{Ru}(\mathrm{SA}) / \mathrm{CeO}_{2}$ and (b) $\mathrm{Ru}(\mathrm{NC}) /$ $\mathrm{CeO}_{2}$ [42]; TEM images of (c) $\mathrm{Rh} / \mathrm{CeO}_{2}-\mathrm{NC}$, (d) $\mathrm{Rh} / \mathrm{CeO}_{2}-\mathrm{NR}$ catalysts [44] (color online)

为 $11.5 \mathrm{~nm}$, 长度为60 150 nm. 不同形貌的 $\mathrm{CeO}_{2}$ 载体会 选择性地暴露出不同晶面，这会影响 $\mathrm{Rh}$ 纳米颗粒的分 散度, 从而影响催化剂的丙二醇重整反应性能.

\subsection{2 共沉淀法}

共沉淀法是一种简便、常用且具有普适性的一步 合成方法, 其反应条件温和, 不需要进行复杂的动力学 过程控制.一般情况下, 它仅需要将金属前驱体、表面 活性剂、沉淀剂混合，然后进行热处理即可得到所需 纳米材料．通过对金属盐、沉淀剂、反应温度和时间 等条件进行调控，可以得到不同组成和结构的贵金属氧化铈负载型催化剂.

例如, $\mathrm{Li}$ 等 ${ }^{[45]}$ 使用 $\mathrm{NaOH}$ 为沉淀剂, 通过一锅共沉 淀法合成了介孔结构的 $\mathrm{Pd}-\mathrm{CeO}_{x}$ 复合材料, 并将其用于 低温 $\mathrm{CO}$ 氧化. 得到的 $\mathrm{Pd}-\mathrm{CeO}_{x}$ 材料具有较高的比表面 积，并且 $\mathrm{Pd}$ 物种在介孔 $\mathrm{CeO}_{x}$ 载体上高度分散，因此在 环境条件下对 $\mathrm{CO}$ 氧化表现出良好的催化活性和稳定 性. 将沉淀法与其他方法相结合，也常用于合成贵金 属-氧化铈复合材料. Derevyannikova等 ${ }^{[46]}$ 以 $\mathrm{KOH}$ 为沉 淀剂，通过共沉淀法制备了不同 $\mathrm{Rh}$ 含量的 $\mathrm{Rh}-\mathrm{CeO}_{2}$ 复 合催化剂，然后在 $450 \sim 1000^{\circ} \mathrm{C}$ 的温度下，对所得 $\mathrm{Rh}$ $\mathrm{CeO}_{2}$ 样品进行烧结处理, 研究烧结过程中催化剂结构
的变化, 以及不同结构对低温 $\mathrm{CO}$ 氧化性能的影响. 结 果表明, $\mathrm{Rh}$ 含量小于 $10 \% \mathrm{wt} \%$ 的样品, 在 $450^{\circ} \mathrm{C}$ 下煅烧, 可得到固溶体相. 当烧结温度高于 $450^{\circ} \mathrm{C}$ 时, 固溶体相 分解, 同时催化剂的催化活性降低.

Chen等 ${ }^{[47]}$ 以 $\mathrm{Na}_{2} \mathrm{CO}_{3}$ 和 $\mathrm{NaOH}$ 为沉淀剂, 通过共沉 淀法合成了 $\mathrm{Pt} / \mathrm{CeO}_{2}$ 复合材料, 金属盐的水解是通过摚 拌过程中向去离子水中缓慢滴加金属盐前驱体溶液来 实现. $\mathrm{Pt}$ 的引入能增强 $\mathrm{CeO}_{2}$ 载体的还原性, 使催化剂表 面 $\mathrm{Ce}^{3+}$ 活性位点显著增多. 此外, 与纯 $\mathrm{CeO}_{2}$ 相比, $\mathrm{Pt} / \mathrm{CeO}_{2}$ 在逆水汽变换反应中表现出优异的催化活性. 总体来说, 共沉淀法是合成多种结构贵金属-氧化铈的 有效方法. 但是, 当贵金属负载量较低时, 它较难保证 所得材料的均一性, 并且共沉淀法在合成核壳结构的 贵金属-氧化铈方面存在一定困难, 这些都有待于我们 进一步研究解决.

\subsection{3 沉积-沉淀法}

沉积-沉淀法是一种广泛使用且有效地用于合成 贵金属-氧化铈负载型催化剂的方法. 它是将贵金属前 驱体溶液与分散有氧化铈载体的悬浊液均匀混合, 在 充分摚拌的条件下，通过调控温度和 $\mathrm{pH}$ 等条件，使贵 金属沉积在氧化铈载体表面. 通过控制贵金属前驱体 的浓度和用量、搅拌和老化时间以及后处理工序等可 以实现对贵金属颗粒尺寸和负载量的调控. 这种方法 对活性组分的利用率较高，同时所得催化剂的尺寸分 布比较均匀.

例如, $\mathrm{Li}^{\text {等 }}{ }^{[48]}$ 以 $\mathrm{NaOH}$ 为沉淀剂, 通过沉积-沉淀法 在 $\mathrm{CeO}_{2}$ 纳米棒 $(\mathrm{NRs})$ 、纳米立方体 $(\mathrm{NCs})$ 、八面体 $(\mathrm{NOs})$ 上负载了 $\mathrm{Au}$ 纳米颗粒, 并且研究了 $\mathrm{CeO}_{2}$ 载体的 形貌对羟甲基糖醛选择性氧化性能的影响. 所得到的 $\mathrm{Au} / \mathrm{CeO}_{2}-\mathrm{NRs} 、 \mathrm{Au} / \mathrm{CeO}_{2}-\mathrm{NCs}$ 和 $\mathrm{Au} / \mathrm{CeO}_{2}-\mathrm{NO}$ 的比表 面积分别为75.8、18.9和 $3.3 \mathrm{~m}^{2} \mathrm{~g}^{-1}$. 其中, $\mathrm{Au} / \mathrm{CeO}_{2}-$ $\mathrm{NRs}$ 对羟甲基糖醛选择氧化效率最高, $\mathrm{TOF}$ 值为 $6.3 \mathrm{~min}^{-1}$.

再如, Liu等 ${ }^{[49]}$ 以聚苯乙烯胶体微球为模板, 先采 用模板法合成了三维有序大孔(3DOM) $\mathrm{CeO}_{2}$, 然后通 过沉积-沉淀法合成了 $3 \mathrm{DOM} \mathrm{Au} / \mathrm{CeO}_{2}$ 复合催化剂. 研 究结果表明, 所制得的 $\mathrm{Au} / \mathrm{CeO}_{2}$ 具有良好的三维有序 大孔结构, $3 \mathrm{DOM} \mathrm{CeO}_{2}$ 的孔径约为 $80 \mathrm{~nm}, \mathrm{Au}$ 纳米颗 粒在其表面分布均匀. $3 \mathrm{DOM} \mathrm{Au} / \mathrm{CeO}_{2}$ 能显著增强甲 醛 $(\mathrm{HCHO})$ 氧化的催化性能, 在 $75^{\circ} \mathrm{C}$ 时 $\mathrm{HCHO}$ 转化率为 
$100 \%$ ，并且 $\mathrm{Au}^{3+}$ 离子对 $\mathrm{HCHO}$ 催化氧化的催化活性高 于金属态的 $\mathrm{Au}^{0}$. Tinoco 等 ${ }^{[50]}$ 通过沉积-沉淀法在 $\mathrm{CeO}_{2}$ 纳米立方体表面负载了 $\mathrm{Au}$ 纳米颗粒, 并研究 $\mathrm{CeO}_{2}$ 表面 纳米结构对其作为负载 $\mathrm{Au}$ 的载体性能的影响. 在 $600^{\circ} \mathrm{C}$ 的氧化处理下, $\mathrm{CeO}_{2}$ 的 $\{100\}$ 表面会部分转化为 $\{111\}$ 纳米切面, 该 $\{111\}$ 纳米切面能显著提高贵金属 在催化剂合成过程中的利用效率. 此外, 在表面重构 的 $\mathrm{CeO}_{2}$ 表面负载的 $\mathrm{Au}$ 催化剂表现出较优异的 $\mathrm{CO}$ 氧化 活性.

\subsection{4 模板法}

模板法分为硬模板法和软模板法，一般用于制备 介孔、中空或核壳结构的贵金属-氧化铈复合材料. 硬 模板法中模板只是作为一个结构支架, 所需要的外壳 材料在其周围原位生成并定向生长成与模板形态互补 的纳米结构. 随后通过高温煆烧或化学腐蚀溶解去除 硬模板. 软模板法与硬模板法类似, 但使用的模板一 般为聚合物、表面活性剂等.

例如, 郑南峰课题组 ${ }^{[51]}$ 通过硬模板法成功合成了 蛋黄结构 $\mathrm{Pd} @ \mathrm{CeO}_{2}$ 纳米催化剂, 如图7 $(\mathrm{a} \sim \mathrm{c})$ 所示. 首 先, 利用Brij 56分子在反胶束体系下制备了均匀、单 分散的 $\mathrm{Pd}-\mathrm{Fe}_{2} \mathrm{O}_{3} @ \mathrm{SiO}_{2}$ 核壳纳米球. 部分 $\mathrm{Fe}_{2} \mathrm{O}_{3}$ 被酸蚀 刻后, 将 $\mathrm{Ce}\left(\mathrm{NO}_{3}\right)_{3}$ 加入到水、乙酸、乙二醇的混合物 中, 并在溶剂热过程中得到 $\mathrm{Pd} @ \mathrm{SiO}_{2}$ 核壳样品, 形成 $\mathrm{Pd} @ \mathrm{SiO}_{2} @ \mathrm{CeO}_{2}$ 纳米球. 通过 $\mathrm{NaOH}$ 选择性去除 $\mathrm{SiO}_{2}$, $\mathrm{Pd} @ \mathrm{SiO}_{2} @ \mathrm{CeO}_{2}$ 纳米球即可被转化为蛋黄结构的 $\mathrm{Pd} @ \mathrm{CeO}_{2}$ 纳米催化剂. 该催化剂具有 $\mathrm{Pd}$ 纳米颗粒 $(<10 \mathrm{~nm})$ 封装在 $\mathrm{CeO}_{2}$ 中的分级结构, 在 $\mathrm{CO}$ 氧化和肉桂 醇氧化成肉桂醛反应中表现出良好的催化活性和稳 定性.

在选定的硬模板表面沉积贵金属纳米颗粒, 然后 再涂覆 $\mathrm{CeO}_{2}$. 去除硬模板后, 将贵金属纳米颗粒封装 在中空 $\mathrm{CeO}_{2}$ 的内部空间中, 可得到中空结构的贵金 属-氧化铈复合材料. 例如, Xia等 ${ }^{[52]}$ 采用等离子体处理 过的聚苯乙烯(PS)纤维作为硬模板, 而处理过的PS的 亲水性表面有利于PVP包覆的Pt纳米颗粒的沉积. 如 图7(d)所示, 由于 $\mathrm{Ce}^{3+}$ 和带负电荷的Pt纳米颗粒之间的 静电相互作用, $\mathrm{Ce}^{3+}$ 阳离子可以很容易地吸附在Pt纳 米颗粒上, 从而促进 $\mathrm{CeO}_{2}$ 纳米晶的成核和生长. 在 $400^{\circ} \mathrm{C}$ 的空气中煅烧去除PS纤维后, 即成功地将Pt纳米 颗粒嵌入到 $\mathrm{CeO}_{2}$ 中空纤维中.

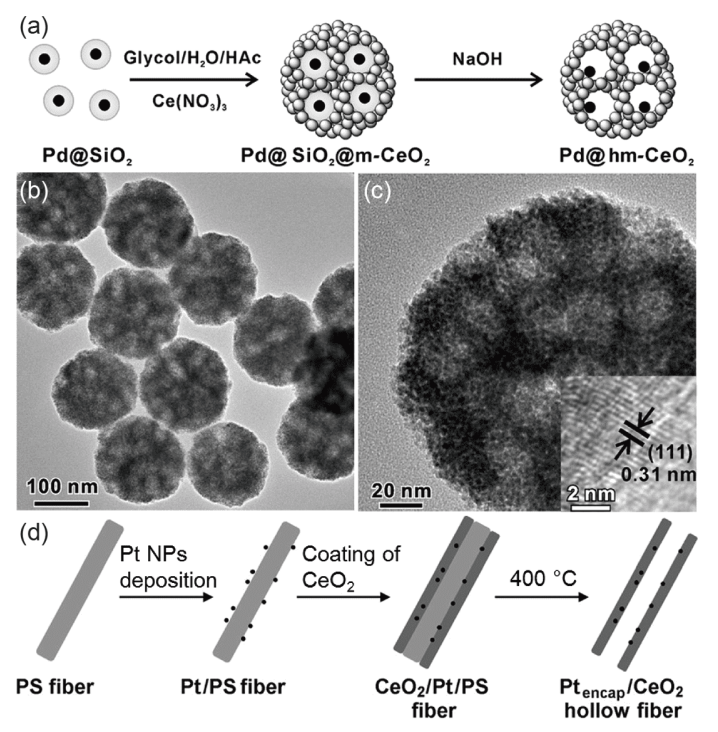

图 $7 \mathrm{Pd} @ \mathrm{CeO}_{2}$ 的合成示意图(a), TEM图像(b), 高分辨电子 显微镜(HRTEM)图像(c) ${ }^{[51]}$; (d) $\mathrm{Pt}-\mathrm{CeO}_{2}$ 纳米空心纤维的合成 示意图 $^{[52]}$

Figure 7 (a) Scheme of the synthetic procedure, (b) TEM, (c) HRTEM images of $\mathrm{Pd} @ \mathrm{CeO}_{2}$ [51]; (d) synthesis procedure of Pt$\mathrm{CeO}_{2}$ hollow fibers [52].

$\mathrm{Du}$ 等 $^{[53]}$ 使用聚合物-模板法合成了 $\mathrm{Pd}-\mathrm{CeO}_{2}$ 纳米 空心球, $\mathrm{Pd}$ 纳米颗粒均匀地分布在 $\mathrm{CeO}_{2}$ 壳层的内部. 由于 $\mathrm{CeO}_{2}$ 纳米晶的稳定作用, 即使在 $700^{\circ} \mathrm{C}$ 炦烧, $\mathrm{Pd}$ 纳 米颗粒也不会发生团聚. $\mathrm{Pd}$ 纳米粒子部分埋在 $\mathrm{CeO}_{2}$ 壳 层中, 因此表面的Pd物种含量较高, 这是由焙烧过程中 $\mathrm{Pd}-\mathrm{CeO}_{2}$ 界面处的电子交换引起的. 这种中空结构的 $\mathrm{Pd}-\mathrm{CeO}_{2}$ 纳米催化剂在水相选择性还原4-硝基苯酚和 气相 $\mathrm{CO}$ 氧化中表现出良好的催化活性和稳定性.

\subsection{5 其他方法}

合成贵金属-氧化铈负载型催化剂的方法有很多, 除了前面所提到的之外, 其他合成方法介绍如下.

本课题组 ${ }^{[54]}$ 先通过水热法合成 $\mathrm{CeO}_{2}$ 纳米线 (NWs), 然后通过超声化学法在 $\mathrm{CeO}_{2} \mathrm{NWs}$ 上负载了不 同尺寸和含量的Pt物种. $\mathrm{CeO}_{2} \mathrm{NWs}$ 的主要暴露晶面是 (110), $\mathrm{Pt}$ 以 $\mathrm{PtO}_{x}$ 团簇的形式存在. 大部分的 $\mathrm{PtO}_{x}$ 位于界 面处, 并受到金属-载体相互作用的影响. 同时, 我们进 一步研究了 $\mathrm{CeO}_{2} \quad \mathrm{NWs}$ 上亚纳米 $\mathrm{PtO}_{x}$ 团簇在低温 $\mathrm{CO}$ 氧 化中的催化活性对界面 $\mathrm{PtO}_{x}$ 位点的局域配位结构敏感 的性质.

$\mathrm{Bal}$ 等 ${ }^{[55]}$ 以十六烷基二甲基溴化氨(CTAB)为表面 活性剂, 聚乙烯吡咯烷酮(PVP)为稳定剂和尺寸控制 
剂, 通过水热法合成了 $\mathrm{Pt}-\mathrm{CeO}_{2}$ 纳米颗粒 $(\mathrm{NPs})$. 其中, $\mathrm{Pt}$ $\mathrm{NPs}$ 的尺寸为 $2 \sim 5 \mathrm{~nm}, \mathrm{CeO}_{2} \mathrm{NPs}$ 载体的尺寸为 $30 \sim 60 \mathrm{~nm}$. 结果表明, 该催化剂在室温下 $\left(25^{\circ} \mathrm{C}\right)$ 有氢分 子存在的水介质中对硝基化合物的化学选择性加氢反 应具有较高的活性. Cui 等 ${ }^{[56]}$ 通过对 $\mathrm{Ce}(\mathrm{OH})_{3}$ 和 $\mathrm{Pd}^{2+}$ 的 原位还原合成了 $\mathrm{Pd} / \mathrm{CeO}_{2}$ 纳米复合材料。在这里, $\mathrm{Ce}\left(\mathrm{NO}_{3}\right)_{3}$ 不仅提供了 $\mathrm{Ce}$ 离子的来源，而且还起到了还 原剂的作用. 通过调节贵金属前驱体溶液的注入速率, 可以调节纳米颗粒的大小.

Simon等 ${ }^{[57]}$ 通过直接液相注射化学气相沉积法 (DIL-CVD) 在 $\mathrm{Si}$ 基底上合成了 $\mathrm{Pt}-\mathrm{CeO}_{2}$ 多孔薄膜材料, 如图 $8(\mathrm{a}, \mathrm{b})$ 所示. 薄膜由直径为 $3 \mathrm{~nm}$ 的 $\mathrm{CeO}_{2}$ 颗粒组成, $\mathrm{Pt}$ 均匀地分散在 $\mathrm{CeO}_{2}$ 膜层中, 并且主要以 $\mathrm{Pt}^{2+}$ 形式存 在. $\mathrm{Pt}$ 前驱体通过预先形成的 $\mathrm{CeO}_{2}$ 薄膜多孔结构扩散 后，在构成薄膜的每个 $\mathrm{CeO}_{2}$ 粒子表面分解，生成均匀 的 $\mathrm{Pt}$ 掺杂 $\mathrm{CeO}_{2}$ 纳米复合材料. 当 $\mathrm{CeO}_{2}$ 颗粒表面的 $\mathrm{Pt}^{2+}$ 位点达到饱和时, 过量的Pt形成金属纳米团簇.

Zhang等 ${ }^{[58]}$ 以 $\mathrm{Na}_{2} \mathrm{PdCl}_{4}$ 为前驱体, 甲醇为牺牲剂, 通过光沉积法在多孔 $\mathrm{CeO}_{2}$ 纳米棒上负载了亚纳米 $\mathrm{Pd}$ 簇 $\left(\mathrm{PdsNC} / \mathrm{PN}-\mathrm{CeO}_{2}\right)$, 如图 $8(\mathrm{c}, \mathrm{d})$ 所示. 在光照射下, $\mathrm{PN}-\mathrm{CeO}_{2}$ 的光生电子可以还原吸附 $\mathrm{Pd}$ 离子. 对于高分 散性Pd催化剂, 在 $\mathrm{PN}-\mathrm{CeO}_{2}$ 的氧空位上优先吸附硝基, 并在 $\mathrm{Pd}$ 上进行氢活化，从而获得较高的催化活性和选 择性.

将贵金属负载在氧化铈表面，可以有效降低贵金 属用量，提高催化性能. 目前常用的制备方法有其自 身独特的优点, 但也存在一些缺点, 如反应过程不易 精确控制、反应条件苛刻，有些方法使用的模板剂或 有机溶剂有毒等, 这些都在一定程度上限制了贵金属氧化铈负载型催化剂的未来实际应用. 因此, 发展操作 更加简单、成本更加低廉、更加安全环保、催化性能 提升更佳的制备该类催化剂的方法，仍有待于进一步 研究.

\section{3 贵金属/氧化铈纳米晶的催化应用}

无论是构建双金属/三金属合金催化剂还是贵金 属-氧化铈负载型催化剂, 我们的主要目的是提高催化 剂性能和降低催化剂成本. 催化反应本质上是一种表 面反应，对催化剂表面的组成、几何结构和电子结构 非常敏感 ${ }^{[59]}$. 通过对催化剂的组成、形貌和结构进行

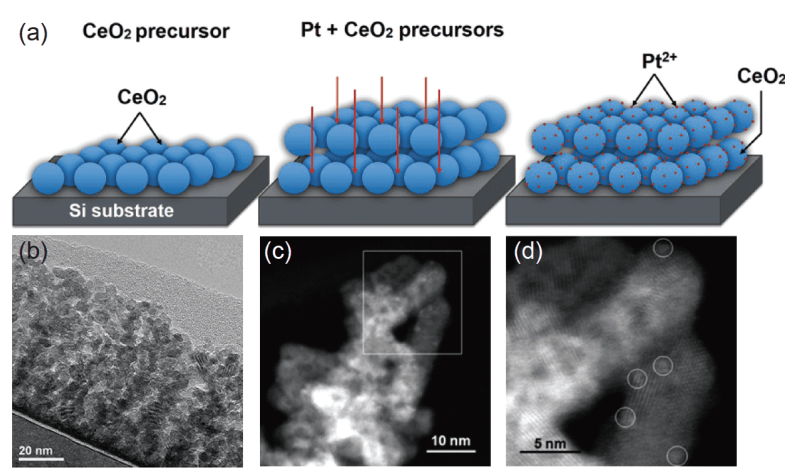

图 $8 \mathrm{Pt}-\mathrm{CeO}_{2}$ 多孔薄膜的生长过程示意图(a)和TEM图像 ${ }^{[57]}$ (b); (c, d) PdsNC/PN-CeO 2 的HAADF-STEM图像 ${ }^{[58]}$ (网络版 彩图)

Figure 8 (a) Growth procedure, (b) TEM image of porous $\mathrm{Pt}-\mathrm{CeO}_{2}$ thin films [57]; (c, d) HAADF-STEM images of $\mathrm{PdsNC} / \mathrm{PN}-\mathrm{CeO}_{2}$ catalysts [58] (color online).

调控, 可以使其展现出最佳的催化性能. 前文中, 我们 详细介绍了实现这两种类型的催化剂形貌和结构可控 合成的主要方法. 接下来, 我们将基于结构与性能的关 系, 介绍它们在催化反应中的应用.

\section{1 双金属/三金属纳米催化剂在多相催化中的 应用}

两种或三种金属元素形成合金之后表面的电子和 几何结构(键长、表面张力等)发生了改变, 使得反应 物及中间体在催化剂表面的吸附能发生了改变, 从而 导致双金属/三金属纳米晶展现出不同寻常的催化活 性及选择性，而被应用于多相催化反应中. 表1总结了 文中列举的双金属/三金属纳米催化剂的合成方法和 催化性能.

\subsection{1 甲醇氧化反应(MOR)}

直接甲醇燃料电池(DMFCs) 由于其燃料洁净环保 和低温快速启动等特点受到人们的广泛关注, 而MOR 是甲醇燃料电池中重要的阳极反应. $\mathrm{Pt}$ 是对 MOR最具 活性的单金属催化剂, 但在相对低电位(例如, $0.6 \mathrm{~V}$ $v s$. RHE)时，其活性会被吸附CO的中毒中间体严重抑 制. 通过将 $\mathrm{Pt}$ 与其他金属复合形成双金属/三金属纳米 晶可以有效提升MOR的催化性能. 目前, 具有多种形 貌的(纳米多面体、纳米笼、纳米线、核壳、树枝状 等)Pt基催化剂, 如PtM (M=Pd、Au、 $\mathrm{Ru} 、 \mathrm{Cu} 、 \mathrm{Ir}$ 等)已 被广泛报道用于催化MOR ${ }^{[60]}$. 
表 1 双金属/三金属纳米催化剂的合成方法、组成结构和催化性能

Table 1 Synthesis, structure and catalytic performances of bimetallic or trimetallic noble metal nanocatalysts

\begin{tabular}{|c|c|c|c|c|c|c|c|c|}
\hline 催化剂 & 合成方法 & 形貌 & 尺寸 (nm) & 组分 & 反应类型 & 性能 & $\begin{array}{l}\text { 影响性能的 } \\
\text { 关键因素 }\end{array}$ & $\begin{array}{l}\text { 参考 } \\
\text { 文献 }\end{array}$ \\
\hline \multirow{3}{*}{$\mathrm{Pd}-\mathrm{Rh}$} & \multirow{3}{*}{ 水热法 } & 中空纳米立方体 & $14.4 \pm 0.6$ & $\mathrm{Pd} / \mathrm{Rh} \approx 1: 2$ & \multirow{3}{*}{ Suzuki交叉偶联 } & TOFs 为 $3 \mathrm{~s}^{-1}$ & \multirow{3}{*}{ 组分和暴露晶面 } & {$[25]$} \\
\hline & & 纳米二十面体 & $16.3 \pm 0.7$ & $\mathrm{Pd} / \mathrm{Rh} \approx 2: 1$ & & $\mathrm{TOFs}$ 为 $1 \mathrm{~s}^{-1}$ & & [25] \\
\hline & & 纳米截断八面体 & $12.1 \pm 0.9$ & $\mathrm{Pd} / \mathrm{Rh} \approx 2: 1$ & & $\mathrm{TOFs}$ 为 $1 \mathrm{~s}^{-1}$ & & {$[25]$} \\
\hline \multirow{2}{*}{ Pt-Ni } & \multirow{2}{*}{ 水热法 } & 纳米立方体 & $20 \pm 2$ & $\mathrm{Pt} / \mathrm{Ni}=38: 62$ & \multirow{2}{*}{ ORR } & $\begin{array}{l}\text { MA: } 0.83 \pm 0.05 \mathrm{~A} \mathrm{mg}_{\mathrm{Pt}^{-1}} \\
\text { SA: } 2.25 \pm 0.10 \mathrm{~mA} \mathrm{~cm}^{-2} \\
\quad \text { 在0.9 V (vs. RHE) }\end{array}$ & \multirow{2}{*}{$\begin{array}{l}\text { 表面组成 } \\
\text { 和形貌 }\end{array}$} & [29] \\
\hline & & 纳米颗粒 & $15 \pm 2$ & $\mathrm{Pt} / \mathrm{Ni}=35: 65$ & & $\begin{array}{l}\text { MA: } 1.01 \pm 0.14 \mathrm{~A} \mathrm{mg}_{\mathrm{Pt}^{-1}} \\
\text { SA: } 2.69 \pm 0.12 \mathrm{~mA} \mathrm{~cm}^{-2} \\
\quad \text { 在0.9 V (vs. RHE) }\end{array}$ & & [29] \\
\hline $\mathrm{PtRuCu}$ & $\begin{array}{l}\text { 溶剂热法+化 } \\
\text { 学刻蚀法 }\end{array}$ & $\begin{array}{l}\text { 菱形十二面体 } \\
\text { 纳米框架结构 }\end{array}$ & $40 \sim 80$ & $\begin{aligned} & \mathrm{Pt} / \mathrm{Ru} / \mathrm{Cu} \\
= & 40.7: 0.3: 59\end{aligned}$ & MOR & $\begin{array}{c}\text { MA: } 0.99 \mathrm{~A} \mathrm{mg}_{\mathrm{Pt}}^{-1} \\
\text { SA: } 2.17 \mathrm{~mA} \mathrm{~cm}{ }^{-2}, \text { 优于 } \\
\text { 商业Pt/C }\end{array}$ & $\begin{array}{l}\text { 组分、比表面 } \\
\text { 积和表面缺陷 }\end{array}$ & [33] \\
\hline $\mathrm{Pt} @ \mathrm{Pd}$ & $\begin{array}{l}\text { Seed-mediated } \\
\text { 生长法 }\end{array}$ & $\begin{array}{c}\text { 核壳结构纳米 } \\
\text { 四面体 }\end{array}$ & 8.3 & $\mathrm{Pt} / \mathrm{Ni} \approx 1: 9$ & HOR & $\begin{array}{c}\text { MA: } 0.19 \mathrm{~A} \mathrm{mg}^{-1} \\
\text { SA: } 0.30 \mathrm{~mA} \mathrm{~cm} \text {, 优于 } \\
\text { 商业Pt/C }\end{array}$ & 暴露晶面(111) & [39] \\
\hline $\mathrm{Au}-\mathrm{Cu}$ & $\begin{array}{c}\text { Galvanic置换 } \\
\text { 法 }\end{array}$ & 空心纳米颗粒 & $\begin{array}{c}50 \sim 100 ; \text { 壁厚 } \\
4.95 \pm 0.81\end{array}$ & $\mathrm{Au} / \mathrm{Cu}=59: 41$ & $\mathrm{CO}_{2} \mathrm{RR}$ & $\begin{array}{c}\text { FE: } 53.3 \% \text { 在 }-0.7 \mathrm{~V} \\
\text { (vs. RHE) }\end{array}$ & $\begin{array}{l}\text { 表面组成和 } \\
\text { 缺陷密度 }\end{array}$ & [40] \\
\hline \multirow{2}{*}{ Pt-Pd } & \multirow{2}{*}{ 水热法 } & 纳米四面体 & $4.9 \pm 0.5$ & $\mathrm{Pt} / \mathrm{Pd}=45: 55$ & \multirow{2}{*}{ MOR } & $\begin{array}{c}J_{\mathrm{f}} / J_{\mathrm{b}}: 1.4 \text {, 循环 } 4000 \text { 圈活 } \\
\text { 性损失 } 18 \%\end{array}$ & \multirow{2}{*}{$\begin{array}{l}\text { (100)和 } \\
(111) \text { 晶面 }\end{array}$} & [61] \\
\hline & & 纳米立方体 & $8.5 \pm 0.8$ & $\mathrm{Pt} / \mathrm{Pd}=46: 54$ & & $\begin{array}{c}J_{\mathrm{f}} / J_{\mathrm{b}}: 2.5, \text { 循环 } 4000 \text { 圈活 } \\
\text { 性损失 } 33 \%\end{array}$ & & [61] \\
\hline Pt-Pd & 水热法 & 纳米二十面体 & $11.2 \pm 0.8$ & $\mathrm{Pt} / \mathrm{Pd}=46: 54$ & MOR & $\begin{array}{c}\text { 活性高于Pt-Pd纳米四 } \\
\text { 面体和商用Pt催化剂 }(\mathrm{Pt} \\
\text { 黑和Pt/C) }\end{array}$ & $\{111\}$ 晶面 & {$[62]$} \\
\hline $\mathrm{Pt}-\mathrm{Cu}$ & 水热+Galvanic & 凹纳米立方体 & $11.2 \pm 1.5$ & $\mathrm{Pt} / \mathrm{Cu}=89: 11$ & & 活性明显高于Pt NCs和 & 表面电子结构 & [41] \\
\hline Pt-Pd-Cu & 置换法 & 凹纳米立方体 & $17.3 \pm 2.9$ & $\begin{array}{c}\mathrm{Pt} / \mathrm{Pd} / \mathrm{Cu} \\
=42: 46: 12\end{array}$ & MOR & 商用 $\mathrm{Pt} / \mathrm{C}$ 催化剂 & 和原子排布 & [41] \\
\hline $\mathrm{Pt}_{3} \mathrm{RhSn}$ & 溶剂热法 & 近球形 & 8.5 & $\mathrm{Pt} / \mathrm{Pd} / \mathrm{Sn}=3: 1: 1$ & EOR & $\begin{array}{c}\text { MA: } 23.4 \mathrm{~mA} \mathrm{mg}_{\mathrm{Pt}}^{-1} \\
\text { SA: } 0.0919 \mathrm{~mA} \mathrm{~cm}^{-2} \text { 在 } \\
0.45 \mathrm{~V} v s . \text { RHE }\end{array}$ & $\begin{array}{l}\text { 组分与表面 } \\
\text { 电子结构 }\end{array}$ & [28] \\
\hline \multirow[b]{2}{*}{ Pt-Pd-Rh } & \multirow[b]{2}{*}{ 水热法 } & 纳米立方体 & 8.8 & $\begin{array}{l}\mathrm{Pt} / \mathrm{Pd} / \mathrm{Ru} \\
=62: 20: 18\end{array}$ & \multirow[b]{2}{*}{ EOR } & $\begin{array}{c}\text { 在 } 0.5 \mathrm{~V} \text { ( } v s . \text { RHE)下活 } \\
\text { 性高, 稳定性最好 }\end{array}$ & \multirow{2}{*}{$\begin{array}{c}\text { 表面成分和 } \\
\text { 暴露晶面 }\end{array}$} & [27] \\
\hline & & 纳米截断八面体 & $11.9 \pm 1.1$ & $\begin{array}{l}\mathrm{Pt} / \mathrm{Pd} / \mathrm{Ru} \\
=39: 33: 28\end{array}$ & & $\begin{array}{c}\text { 在 } 0.5 \mathrm{~V}(v s . \mathrm{RHE}) \text { 下活 } \\
\text { 性高, 对 } \mathrm{CO}_{2} \text { 的选择性 } \\
\text { 最高 }\end{array}$ & & {$[27]$} \\
\hline Pt-Ni-Rh & 湿化学法 & 纳米八面体 & $7.7 \pm 1.2$ & $\begin{array}{l}\mathrm{Pt} / \mathrm{Ni} / \mathrm{Ru} \\
=77: 20: 3\end{array}$ & EOR & $\begin{array}{l}\text { 最低开路电压为 } 0.1 \mathrm{~V} \\
\text { (vs. RHE), 打断 } \mathrm{C}-\mathrm{C} \text { 键 }\end{array}$ & 三元表面位点 & [65] \\
\hline \multirow{3}{*}{ Ir-Pd } & \multirow{3}{*}{ 溶剂热法 } & 纳米空心球 & $7.3 \pm 1.0$ & $\mathrm{Ir} / \mathrm{Pd}=40: 60$ & \multirow{3}{*}{ OER } & $\begin{array}{c}\text { MA: } 44.4 \mathrm{~A} \mathrm{~g}^{-1} \text {, 酸性 } \\
\text { 介质 }\end{array}$ & \multirow{3}{*}{$\begin{array}{c}\text { 表面合金效应 } \\
\text { 和氧化效应 }\end{array}$} & [31] \\
\hline & & 纳米线 & $2.9 \pm 0.4$ & $\mathrm{Ir} / \mathrm{Pd}=42: 58$ & & $\begin{array}{c}\text { MA: } 74.9 \mathrm{~A} \mathrm{~g}^{-1} \text {, 酸性 } \\
\text { 介质 }\end{array}$ & & [31] \\
\hline & & 纳米四面体 & $5.7 \pm 0.8$ & $\mathrm{Ir} / \mathrm{Pd}=21: 79$ & & 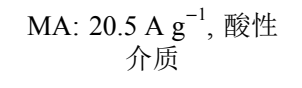 & & [31] \\
\hline Ir-Co-Ni & 离子刻蚀法 & $\begin{array}{c}\text { 多孔空心纳米 } \\
\text { 颗粒 }\end{array}$ & 62 & $\mathrm{Ir} / \mathrm{Co} / \mathrm{Ni}=1: 1: 1$ & OER & $\begin{array}{c}\text { 低开路电压: } 1.46 \mathrm{~V}(v s . \\
\mathrm{RHE} \text { ), TOF为 } 0.26 \mathrm{~s}^{-1} \\
\text { (高于商用 Ir) }\end{array}$ & $\begin{array}{c}3 \mathrm{~d} \text { 过渡金属的 } \\
\text { 配位效应 }\end{array}$ & [67] \\
\hline
\end{tabular}


中国科学: 化学 2020 年 第 50 卷 第 11 期

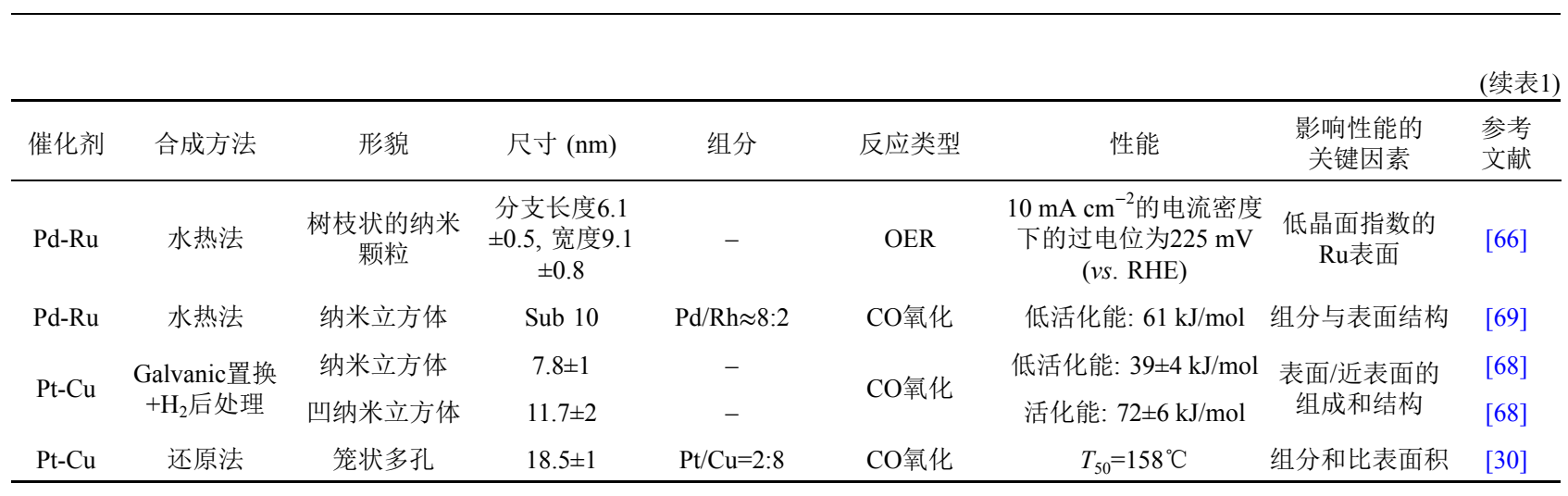

本课题组 ${ }^{[61]}$ 以小离子 $\left(\mathrm{Br}^{-}\right.$或 $\left.\mathrm{I}^{-}\right)$和 $\mathrm{C}_{2} \mathrm{O}_{4}{ }^{2-}$ 为(100)或 (111) 晶面选择剂, PVP为包覆剂, 通过一锅水热法合成 了小于 $10 \mathrm{~nm}$ 的单分散的单晶Pt-Pd纳米四面体(NTs) 和纳米立方体(NCs), 如图9所示. 其中, Pt-Pd NTs的暴 露晶面是(111), Pt-Pd NCs的暴露晶面是(100). 由于 MOR在(100)和(111)表面上的反应途径不同, Pt-Pd NCs在MOR过程中表现出较高的活性, 而Pt-Pd NTs则 表现出较好的稳定性. 在此基础上, 本课题组 ${ }^{[62]}$ 通过 一锅水热法合成了具有多个 $\{111\}$ 孪晶面包围的双金 属Pt-Pd纳米二十面体(NIs), 其形貌选择性高达 $82 \%$. 与 $\{111\}$ 包裹的Pt-Pd纳米四面体以及用于甲醇氧化的 商用 $\mathrm{Pt}$ 催化剂 $(\mathrm{Pt}$ 黑和 $\mathrm{Pt} / \mathrm{C})$ 相比, $\{111\}$ 包裹的NIs 具有 更好的MOR催化活性. 从中可以看出, 暴露晶面对催 化剂的催化性能影响非常大, 通过对双金属/三金属纳 米晶的暴露晶面进行调控, 可以使其暴露出更多的高 反应活性中心, 从而实现催化剂的高活性和选择性. 本课题组 ${ }^{[41]}$ 还合成了由 $\{h k 0\}$ 高指数台阶面包围的Pt$\mathrm{Cu}$ 双金属凹纳米立方体 $(\mathrm{CNCs})$ 和Pt-Pd-Cu三金属凹纳 米立方体. 经紫外线/臭氧清洗后, 这些 $\mathrm{CNCs}$ 在甲醇氧 化中的比活性明显高于 $\mathrm{Pt} \mathrm{NCs}$ 和商用 $\mathrm{Pt} / \mathrm{C}$ 催化剂. 我 们发现表面电子结构 $(\mathrm{Pt}$ 与 $\mathrm{Cu}$ 与 $\mathrm{Pd}$ 的合金化)和表面原 子排布(高指数晶面的暴露)的调变是提高铂基纳米晶 催化MOR性能的有效途径.

负载材料的表面结构对负载催化剂的性能有很大 的影响. 金属基体的表面结构可以影响另一种金属在 其上的沉积 ${ }^{[63]}$, 导致双金属纳米催化剂电子结构的改 变, 从而影响催化剂的性能. 本课题组 ${ }^{[64]}$ 以三种不同 形貌(三角板(TPs)、帽式楞柱 (CCs) 和纳米球 (NSs)) 和 表面结构的 $\mathrm{Ru}$ 纳米晶为载体, 通过水相吸附-还原方法 沉积Pt粒子, 得到了 $\mathrm{Pt} / \mathrm{Ru}$ 双金属纳米晶. 通过测试这 些复合催化剂对MOR的催化活性和稳定性, 建立了 $\mathrm{Ru}$
纳米晶表面结构与Pt粒子催化性能的关系. Pt/Ru TPs/C 比 $\mathrm{Pt} / \mathrm{Ru} \mathrm{CCs} / \mathrm{C}$ 表现出更高的催化活性和稳定性, 说明 在MOR过程中, $\mathrm{Ru}$ 的密排面比Ru的高指数面更适合 作为沉积 $\mathrm{Pt}$ 颗粒的基底. $\mathrm{Pt}$ 纳米颗粒与 $\mathrm{Ru}$ 纳米球在 $\mathrm{Pt} / \mathrm{Ru} \mathrm{NSs} / \mathrm{C}$ 中形成了自支撑网络结构; 与 $\mathrm{Pt} / \mathrm{Ru} \mathrm{CCs} / \mathrm{C}$ 相比, 其增加了Pt的分散程度, 从而表现出更高的催化 活性和稳定性. 从该工作中, 我们可以更好地把握如何 使用形貌良好控制的金属纳米晶来研究催化反应中载 体的表面结构效应.

\subsection{2 乙醇氧化反应 $(E O R)$}

直接乙醇燃料电池(DEFCs) 由于其能量密度高、 易于储存和运输以及对环境的低影响, 在节能和可持 续能源系统中具有巨大的应用潜力. 然而, 由于其阳 极反应动力学缓慢, 催化剂的活性、选择性和耐久性 较差, 阻碍了DEFCs的发展. 乙醇氧化反应(EOR)的迟 缓动力学对设计和合成高性能的多组分纳米催化剂提 出了挑战. 用于EOR的催化剂由单金属Pt催化剂、二 元Pt基催化剂演变为三元Pt基催化剂, 表明 $\mathrm{EOR}$ 不同 的基元反应需要多个活性位点协同工作. 并且在Pt表 面添加活性位点 (主要为Rh或 Ir位点) 有助于解决提高 EOR性能的两个主要问题: $\mathrm{C}-\mathrm{C}$ 键裂解以获得高选择 性的 $\mathrm{CO}_{2}$ 产物, 以及去除 $\mathrm{C} 1$ 中间体以获得高活性.

本课题组 ${ }^{[28]}$ 以DMF为形貌与结构调控剂, 通过一 步溶剂热法合成了一系列几何结构(近球形)和晶粒尺

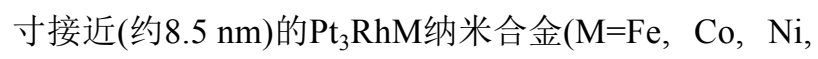
$\mathrm{Cu} ; \mathrm{Ga}, \mathrm{In}, \mathrm{Sn}, \mathrm{Pb})$. 在低电位条件下, 主族金属在提 高EOR的活性和 $\mathrm{CO}_{2}$ 选择性方面优于过渡金属. 特别 地, 在酸性条件下, $\mathrm{Pt}_{3} \mathrm{RhSn} / \mathrm{C}$ 在 $0.45 \mathrm{~V}(v s$. RHE) 的 $\mathrm{EOR}$ 比活性和质量活性相较于商业 $\mathrm{Pt} / \mathrm{C}$ 分别提高了 67 倍和7倍. 通过DFT计算发现, EOR活性与氧和碳的结 

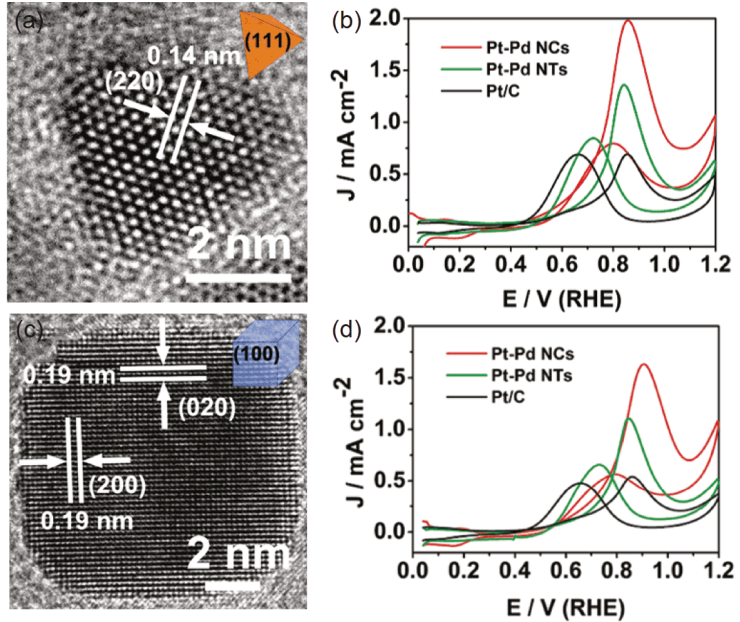

图 9 单个Pt-Pd NT (a), Pt-Pd NC (c)的HRTEM图像; Pt-Pd $\mathrm{NCs}$, NTs 和Pt/C的稳定 $\mathrm{CV}$ 曲线(b)和循环 4000 圈之后的 $\mathrm{CV}$ 曲线 ${ }^{[61]}(\mathrm{d})$ (网络版彩图)

Figure 9 HRTEM images of single (a) Pt-Pd NT and (c) Pt-Pd NC, (b) stable $\mathrm{CV}$ curves and (d) $\mathrm{CV}$ curves obtained after 4000 cycles of Pt-Pd NCs, Pt-Pd NTs, and Pt/C [61] (color online).

合能之和 $\left(E_{\mathrm{O}}+E_{\mathrm{C}}\right)$ 之间呈“火山形”关系. 在上述催化剂 中, $\mathrm{Pt}_{3} \mathrm{RhSn} / \mathrm{C}$ 催化剂的 $E_{\mathrm{O}}+E_{\mathrm{C}}$ 值是最佳的, 对稳定中间 体的吸附强度适中. 此外, $\mathrm{CO}_{2}$ 的选择性与氧和水的结 合能之和 $\left(E_{\mathrm{O}}+E_{\mathrm{H}_{2} \mathrm{O}}\right)$ 也呈线性相关，这也与所制备催化 剂的实验结果非常吻合.

催化剂的形貌、尺寸和暴露晶面也会对EOR性能 产生影响. 例如，Erini等 ${ }^{[65]}$ 报道了一种使用湿化学方 法合成的形貌可控的PtNiRh纳米八面体电催化剂. 与 球形纳米晶相比，其是由富Pt框架、富 $\mathrm{Ni}$ 晶面和 $\mathrm{Rh}$ 聚 集体所构成，从而表现出更好的EOR性能. 这表明形 成特定活性位点的八面体表面具有高效切断 $\mathrm{C}-\mathrm{C}$ 键的 能力. 再如, 本课题组 ${ }^{[27]}$ 利用水热法, 通过调节金属前 体的还原趋势，制备了具有不同组成的暴露(111)晶面 的Pt-Pd-Rh纳米截角八面体(NTOs)和暴露(100)晶面的 纳米立方体(NCs), 如图10所示. 结合DFT计算模拟, 我 们篎选出了 $\mathrm{Pt}_{3} \mathrm{PdRh}$ NTOs、PtPdRh NTOs和 $8.8 \mathrm{~nm}$ PtPdRh NCs-200这三种催化剂, 它们的催化性能最好; 在0.5 V (vs. NHE)条件下具有最高的EOR活性(是商业 $\mathrm{Pt}$ 黑的5倍). 在这些催化剂中, PtPdRh NTOs在 $0.5 \mathrm{~V}$ 时 对 $\mathrm{CO}_{2}$ 的选择性最高, 在极低电位 $(0.35 \mathrm{~V})$ 时具有优异 的乙醇完全氧化能力; 而 $8.8 \mathrm{~nm}$ PtPdRh NCs-200的稳 定性最好. 研究结果表明, 形貌和表面组成与三种金属 的协同催化作用相关，这也验证了形貌和表面组成对

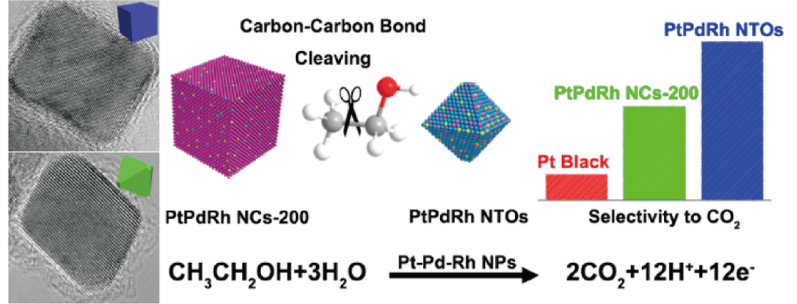

图 10 Pt-Pd-Rh NCs和NTOs的TEM图、结构图、EOR反 应和催化性能 ${ }^{[27]}$ (网络版彩图)

Figure 10 TEM images, structures and EOR catalytic performances of Pt-Pd-Rh NCs and NTOs [27] (color online).

Pt-Pd-Rh纳米晶提高EOR性能的影响. 结合原位傅里 叶变换红外光谱(FTIR)分析结果, 发现提高 $\mathrm{C}-\mathrm{C}$ 键裂 解至低电位能力的关键因素是合适的表面成分和暴露 晶面。

\subsection{3 氧析出反应(OER)}

OER在电化学能源装置的电化学反应过程中是一 种重要的阳极半反应. 与阴极反应相比, 阳极的OER一 般涉及多步电子转移路径, 以及以质子作为载流子时 需要较高的过电位. OER的迟缓动力学对高性能催化 剂的设计提出了更高的要求, 目前钓 $\left(\mathrm{Ru} / \mathrm{RuO}_{2}\right)$ 和铱 $\left(\mathrm{Ir} / \mathrm{IrO}_{2}\right)$ 被认为是两种最高效的OER催化剂体系. 然而 $\mathrm{RuO}_{2}$ 的稳定性较差，单金属组分的 $\mathrm{Ru}$ 基或Ir基催化剂 性能的提升被限制于调控形貌以增加活性位点的数量 和改变金属-载体相互作用等. 目前, 大量的研究集中 于双金属/三金属纳米催化剂, 可以通过更多的方法和 途径(如引入尺寸、形貌、晶相、组分、第二金属的 掺杂效应等)来调控催化剂的OER性能.

本课题组 ${ }^{[31]}$ 通过一步溶剂热法合成了具有纳米 空心球(NHSs)、纳米线(NWs)和纳米四面体(NTs)结 构的Ir-Pd合金. 电化学研究表明Ir-Pd合金纳米催化剂 在酸性和碱性介质中对OER活性均表现出表面微观结 构敏感的增强效应. 在酸性和碱性介质中, 当过电位为 $0.25 \mathrm{~V}$ 时, Ir-Pd NWs和NTs的质量活性比商用 Ir/C催化 剂高5倍以上. XPS分析结果表明, 在Ir-Pd纳米催化剂 的表面缺陷处产生的表面 $\operatorname{Ir}(\mathrm{VI})$ 氧化物可能是影响 OER性能的关键中间体. 类似地, Gloag 等 ${ }^{[66]}$ 利用立方 相核-六方相分支结构的机制，合成了具有面心立方 (fcc)-Pd核和六方(hcp)-Ru分支的树枝状纳米颗粒. 在 油胺表面分散剂作用下, Ru可以在分支表面形成低指 
数晶面. 这种Pd-Ru树枝状纳米颗粒的OER几何电流密 度在 15 min后降低 $33 \%$, 比球形Pd-Ru NPs更稳定，其 稳定性的增强主要归因于具有低晶面指数的 $\mathrm{Ru}$ 表面.

双金属/三金属纳米晶的组分会影响催化剂表面 的原子排布和化学价态等, 造成表面几何结构和电子 结构的变化, 从而影响催化剂的OER性能. Feng 等 ${ }^{[67]}$ 利用 $\mathrm{Fe}^{3+}$ 离子蚀刻 $\mathrm{IrCo} 、 \mathrm{IrNi}$ 和IrCoNi的固体颗粒合成 了 IrCo、IrNi和IrCoNi多孔空心纳米颗粒(PHNCs), 如 图11所示. 他们通过比较 $\mathrm{IrCoNi}$ 和Ir表面上计算出的 $\mathrm{OH} 、 \mathrm{O}$ 和OOH的结合能，证明了OER活性的增强依赖 于所掺杂的过渡金属. 掺杂过渡金属后, Ir表面上氧物 种的稳定结合变弱, 而结合能的变化顺序为 $\mathrm{IrCoNi}<\mathrm{Ir}-$ $\mathrm{Co}<\mathrm{IrNi}<\mathrm{Ir}$ ，其中，多孔空心结构的IrCoNi表现出最好 的OER活性.

\subsubsection{CO氧化反应}

$\mathrm{CO}$ 是汽车尾气和工业废气中的主要污染物, 净化 它的最直接有效的方法就是催化氧化法, 将其转化为 相对无害的 $\mathrm{CO}_{2}$ (反应式: $\mathrm{CO}+\mathrm{O}_{2} \rightarrow \mathrm{CO}_{2}$ ). $\mathrm{CO}$ 氧化反应 简单且有代表性，一般常被用作探针反应来评估催化 剂的性能. 此外, 低温 $\mathrm{CO}$ 氧化在汽车尾气净化、 $\mathrm{CO}$ 气 体传感器、防毒面具等方面具有重要的实际应用价 值. 贵金属以其自身优异的催化活性被认为是 $\mathrm{CO}$ 氧化 反应的首选催化剂, 根据其结构特点可分为非负载型 和负载型催化剂. 本节主要介绍了非负载型的双金属/ 三金属纳米催化剂.

双金属/三金属纳米催化剂的催化性能主要是由 催化剂表面甚至是亚表面的几何结构和电子结构决定 的. 例如, Shan等 ${ }^{[68]}$ 通过在反应气体中后处理的方法 得到了 $\mathrm{Pt}-\mathrm{Cu}$ 常规纳米立方体 $(\mathrm{RNC})$ 和凹纳米立方体 (CNC). Pt-Cu RNC和Pt-Cu CNC催化剂新形成的表面 结构与合成后反应中所使用的反应气体强烈相关.

如图12所示, $\mathrm{Pt}-\mathrm{Cu} \mathrm{RNC}$ 在 $200^{\circ} \mathrm{C}$ 的 $\mathrm{H}_{2}$ 中反应生成 一种亚表面合金(Pt-Cu RNC- $\left.\mathrm{H}_{2}\right)$ ，包括Pt表皮层、富 $\mathrm{Cu}$ 的次表面和富Pt的深层. 并且, Pt-Cu RNC- $\mathrm{H}_{2}$ 在CO 氧化中表现出更高的催化活性, 其活化能为 $39 \pm 4 \mathrm{~kJ} / \mathrm{mol}$, 远低于Pt-Cu RNC $(128 \pm 7 \mathrm{~kJ} / \mathrm{mol})$.

一般来说，双金属纳米晶的组分会影响催化剂表 面的原子排布和化学价态等, 造成表面几何结构和电 子结构的变化, 从而影响催化剂的性能. 本课题组 ${ }^{[69]}$ 采用一锅水热法制备了组分不同的小于 $10 \mathrm{~nm}$ 的Pd-

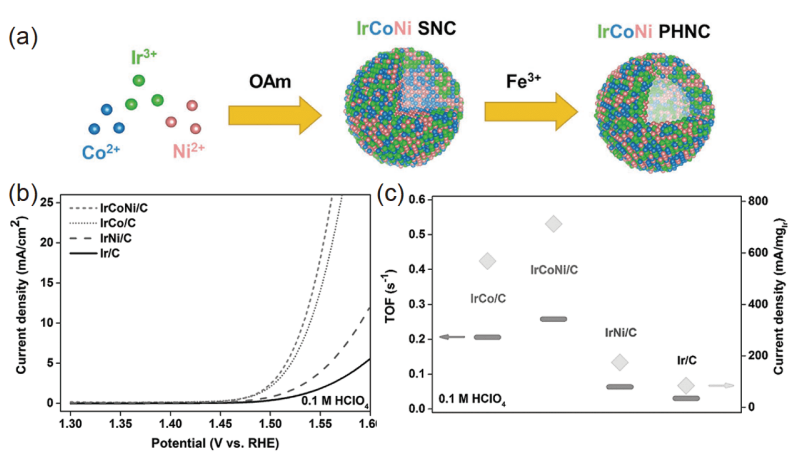

图 $11 \mathrm{IrCoNi}$ PHNCs的合成示意图(a), OER性能图(b), 转 换频率(TOF)和OER电流密度(c) ${ }^{[67]}$ (网络版彩图)

Figure 11 (a) Scheme of the synthetic procedure for IrCoNi PHNCs, (b) OER activities, (c) turnover frequency (TOF) and current densities of OER for the synthesized catalysts [67] (color online).
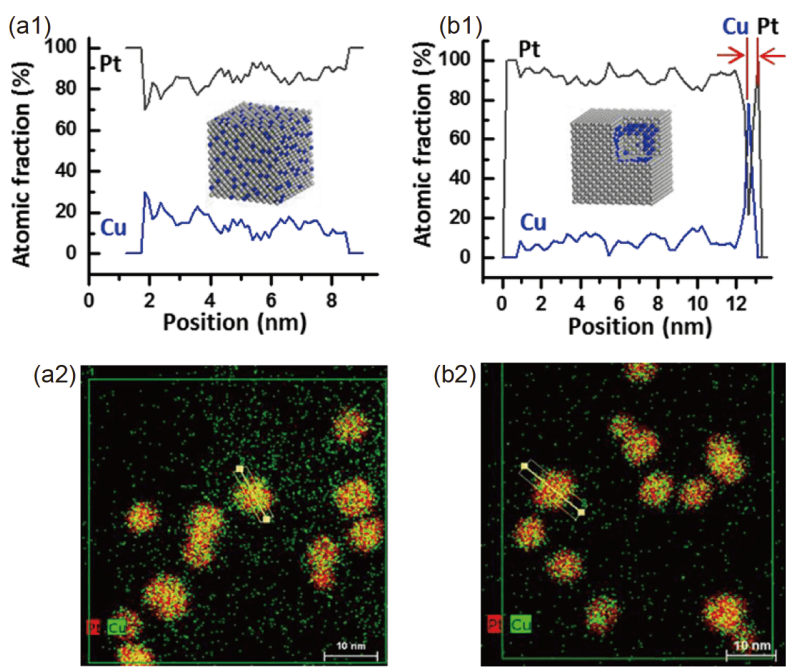

图 $12 \mathrm{Pt}-\mathrm{Cu}$ 纳米催化剂的能量分散X射线图谱(a)和元素分 布图(b). (a1, a2) Pt-Cu RNC, (b1, b2) Pt-Cu RNC- $\mathrm{H}_{2}{ }^{[68]}$ (网络 版彩图)

Figure 12 Energy-dispersive X-ray spectra (a) and mapping of $\mathrm{Pt}-\mathrm{Cu}$ nanocatalysts (b). (a1, a2) Pt-Cu RNC-as synthesized, (b1, b2) Pt-Cu RNC-H ${ }_{2}$ [68] (color online).

$\mathrm{Rh}$ 纳米立方体(包括 $\mathrm{Pd}_{0.8} \mathrm{Rh}_{0.2} 、 \mathrm{Pd}_{0.6} \mathrm{Rh}_{0.4}$ 和 $\mathrm{Pd}_{0.2} \mathrm{Rh}_{0.8}$ ), 它们的形貌和尺寸相似, 暴露晶面均是(100)晶面, 这 样可以排除可能的结构影响. 在 $\mathrm{CO}$ 氧化过程中, 使用 $\mathrm{H}_{2}$ 和 $\mathrm{H}_{2}-\mathrm{O}_{2}$ 进行预处理后, $\mathrm{Pd}_{0.8} \mathrm{Rh}_{0.2} \mathrm{NCs}$ 和 $\mathrm{Pd}_{0.6} \mathrm{Rh}_{0.4}$ $\mathrm{NCs}$ 的表面可分别形成双金属位点和金属/氧化物界 面, 而对于 $\mathrm{Pd}_{0.2} \mathrm{Rh}_{0.8} \mathrm{NCs}$, 反应过程中只观察到合金位 点. 并且只有 $\mathrm{Pd}_{0.6} \mathrm{Rh}_{0.4} \mathrm{NCs}$ 会发生表面偏析，而 $\mathrm{Pd}_{0.8} \mathrm{Rh}_{0.2} \mathrm{NCs}$ 和 $\mathrm{Pd}_{0.2} \mathrm{Rh}_{0.8} \quad \mathrm{NCs}$ 的表面成分保持不变. 
结果表明不同组分的Pd-Rh NCs在催化反应过程中的 表面结构变化不同，从而表现出不同的催化性能. 例 如, 本课题组 ${ }^{[30]}$ 在油胺中还原金属前驱体得到了笼状 多孔Pt-Cu和树枝状多孔Pt-Zn、Pt-Ni纳米颗粒，其中， 多孔Pt-Cu纳米颗粒表现出最优异的 $\mathrm{CO}$ 氧化性能. 这 也进一步说明了组分对催化性能的重要影响.

\section{2 贵金属-氧化铈负载型催化剂的应用}

贵金属可以通过金属-载体相互作用(SMSI)等负 载在氧化铈表面，许多催化反应均倾向于发生在贵金 属与氧化铈之间的界面. 贵金属-氧化铈负载型催化剂 不仅可以将贵金属和氧化铈的功能结合起来，而且与 单组分相比具有独特的协同催化性能. 通过SMSI和尺 寸效应等能够有效提高催化剂的活性、选择性和稳定 性，因而其在低温 $\mathrm{CO}$ 氧化反应、水汽转化、 $\mathrm{CO}_{2}$ 甲烷 化、甲烷催化燃烧、有机液相的选择性氧化还原等反 应中有广泛的应用. 表2总结了常见贵金属-氧化铈负 载型催化剂的合成方法、结构特征以及催化性能.

\subsubsection{CO催化氧化}

催化氧化是控制空气中对人体健康和环境均有危 害的CO污染物的重要途径. 目前, 不同结构和性质的 贵金属-氧化铈复合材料被广泛用于 $\mathrm{CO}$ 氧化.

例如，严纯华课题组 ${ }^{[70]}$ 采用溶剂热法制备了 $\mathrm{Pt}$ 嵌 套的多孔 $\mathrm{CeO}_{2}$ 空心球 $\left(\mathrm{Pt} / \mathrm{CeO}_{2} \mathrm{HS}\right)$ 复合材料. 如图13 $(\mathrm{a}, \mathrm{b})$ 所示, $\mathrm{Pt} / \mathrm{CeO}_{2} \mathrm{HS}$ 复合材料是通过自组装还原-奥 斯特瓦尔德熟化过程得到的, 其中Pt纳米颗粒(Pt NPs) 嵌入 $\mathrm{CeO}_{2}$ 中孔空心球体. $\mathrm{Pt} / \mathrm{CeO}_{2} \mathrm{HS}$ 对 $\mathrm{CO}$ 氧化反应表 现出优异的催化活性和稳定性, CO转化率为 $100 \%$ 的 温度 $\left(T_{100}\right)$ 为 $155^{\circ} \mathrm{C}$, 并且在 $152^{\circ} \mathrm{C}$ 下应 $12 \mathrm{~h}$ 后的 $\mathrm{CO}$ 转 化率仍然维持在 $78 \%$ 左右.

值得注意的是，在分子水平上理解贵金属-氧化铈 负载型催化剂的结构效应对于设计和开发高性能、低 成本催化剂非常重要. 本课题组 ${ }^{[54]}$ 以低温 $\mathrm{CO}$ 氧化为探 针反应研究了局域配位结构对 $\mathrm{CeO}_{2}$ 纳米线 $(\mathrm{NWs})$ 上亚 纳米 $\mathrm{PtO}_{x}$ 团簇的催化活性的影响. $\mathrm{CeO}_{2} \mathrm{NWs}$ 的主要暴 露晶面是(110), $\mathrm{Pt}$ 是以 $\mathrm{PtO}_{x}$ 团簇的形式存在. 通过原位 光谱表征和DFT计算, 研究了不同的亚纳米 $\mathrm{PtO}_{x} / \mathrm{CeO}_{2}$ $\mathrm{NWs}$ 的局部配位结构, 以揭示低温 $\mathrm{CO}$ 氧化过程中 $\mathrm{PtO}_{x}$ 位点的反应活性与其局域结构之间的关系. 结果表明, 虽然亚纳米尺度上高度分散的Pt位点可以提供更多的
可接近活性位点，但由于周围氧的增加可能导致Pt原 子过于稳定，一些Pt位点并没有表现出高的 $\mathrm{CO}$ 氧化活 性. 如图13(c)所示，氧化态Pt位点对 CO中间体的强吸 附, 以及 $\mathrm{PtO}_{x}$ 团簇中具有更高界面晶格氧配位数的 $(\mathrm{CN}(\mathrm{Pt}-\mathrm{O}))$ 的Pt位点比例的增加, 会导致有效活性位点 减少和催化性能的下降. 而亚纳米 $\mathrm{PtO}_{x} / \mathrm{CeO}_{2} \mathrm{NWs}$ 的 $\mathrm{CN}(\mathrm{Pt}-\mathrm{O})$ 值相对较小, 因此可以将活性提高约 $1 / 2$ 个数 量级.

贵金属的负载量及其所处的环境与其催化性能也 具有较强的关联性 ${ }^{[46,71]}$. 例如, Zhao 等 ${ }^{[71]}$ 报道了 $\mathrm{H}_{2} \mathrm{O}$ 对 $\mathrm{CO}$ 氧化具有显著的活性位点依赖性促进作用. 在 $\mathrm{H}_{2} \mathrm{O}$ 存在的情况下, $\mathrm{CeO}_{2}$ 负载的 $\mathrm{Au}$ 单原子催化剂对 $\mathrm{CO}$ 的 氧化促进作用比 $\mathrm{CeO}_{2}$ 负载的 $\mathrm{Au}$ 纳米颗粒催化剂高两 个数量级. 理论研究表明, $\mathrm{H}_{2} \mathrm{O}$ 对 $\mathrm{Au}$ 单原子样品的显著 促进作用源于其独特的局域原子结构和电子性质。带 正电荷的非零价的 $\mathrm{Au}$ 原子具有多种氧化态(如 $\mathrm{Au}(\mathrm{III})$ 、 $\mathrm{Au}(\mathrm{II}) 、 \mathrm{Au}(\mathrm{I}))$, 它作为电子受体可以为存在 $\mathrm{OH}-$ 基团 (或水分子)参与的 $\mathrm{CO}+\mathrm{OH}$ 反应途径提供更有效的 通道.

\subsection{2 $\mathrm{CO}_{2}$ 甲烷化}

$\mathrm{CO}_{2}$ 甲烷化是合成天然气、合成氨原料气的提纯 和净化以及减少 $\mathrm{CO}_{2}$ 排放的重要反应过程，能够实现 $\mathrm{CO}_{2}$ 的资源化和循环利用. 铈基材料负载后过渡金属, 如 $\mathrm{Ni} 、 \mathrm{Co}$ 和 $\mathrm{Ru}$ 等, 是重要的甲烷化催化剂 ${ }^{[72]}$. 氧化铈 由于其可控的形貌、独特的结构(尤其是表面氧缺陷) 而被广泛用作还原性氧化物载体，在其表面负载贵金 属, 能够显著提高 $\mathrm{CO}_{2}$ 甲烷化性能.

强金属-载体相互作用 $(\mathrm{SMSI})$ 可以在 $\mathrm{CO}_{2}$ 甲烷化反 应中观察到，并且贵金属颗粒尺寸会对其造成显著影 响. 本课题组 ${ }^{[42]}$ 在 $\mathrm{CeO}_{2}$ 纳米线上负载了 $\mathrm{Ru}$ 单原子 $(\mathrm{SA}) 、 \mathrm{Ru}$ 团簇 $(\mathrm{NC})$ 和 $\mathrm{Ru}$ 纳米颗粒 $(\mathrm{NPs})$, 并测试了它 们的 $\mathrm{CO}_{2}$ 甲烷化性能. 其中, 纳米团簇表现出最优异的 低温 $\mathrm{CO}_{2}$ 甲烷化活性，选择性为 $98 \% \sim 100 \%$; 在 $190^{\circ} \mathrm{C}$ 时 的转换频率(TOF)为 $7.41 \times 10^{-3} \mathrm{~s}^{-1}$. 这种反应活性的差 异主要是由SMSI以及H溢流效应的竞争作用共同决 定的，如图14所示. 当金属颗粒尺寸从NPs减小到SAs 时, 由于 $\mathrm{Ru}-\mathrm{O}-\mathrm{Ce}$ 键数增加, SMSI效应增强, 改善了 $\mathrm{Ru}$ 到 $\mathrm{CeO}_{2}$ 之间的电子转移. 这导致 $\mathrm{Ru}(\mathrm{SAs}) / \mathrm{CeO}_{2}$ 的正电 荷低于 $\mathrm{CO}$, 从而促进羰基的活化. 但与此同时，粒径 的减小削弱了 $\mathrm{Ru}-\mathrm{H}$ 键, 从而导致 $\mathrm{H}$ 溢出. 结果表明, $\mathrm{H}$ 
表 2 常见贵金属-氧化铈纳米催化剂的合成方法、组成结构和催化性能

Table 2 Synthesis, structure and catalytic performances of cerium oxide supported noble metal nanocatalysts

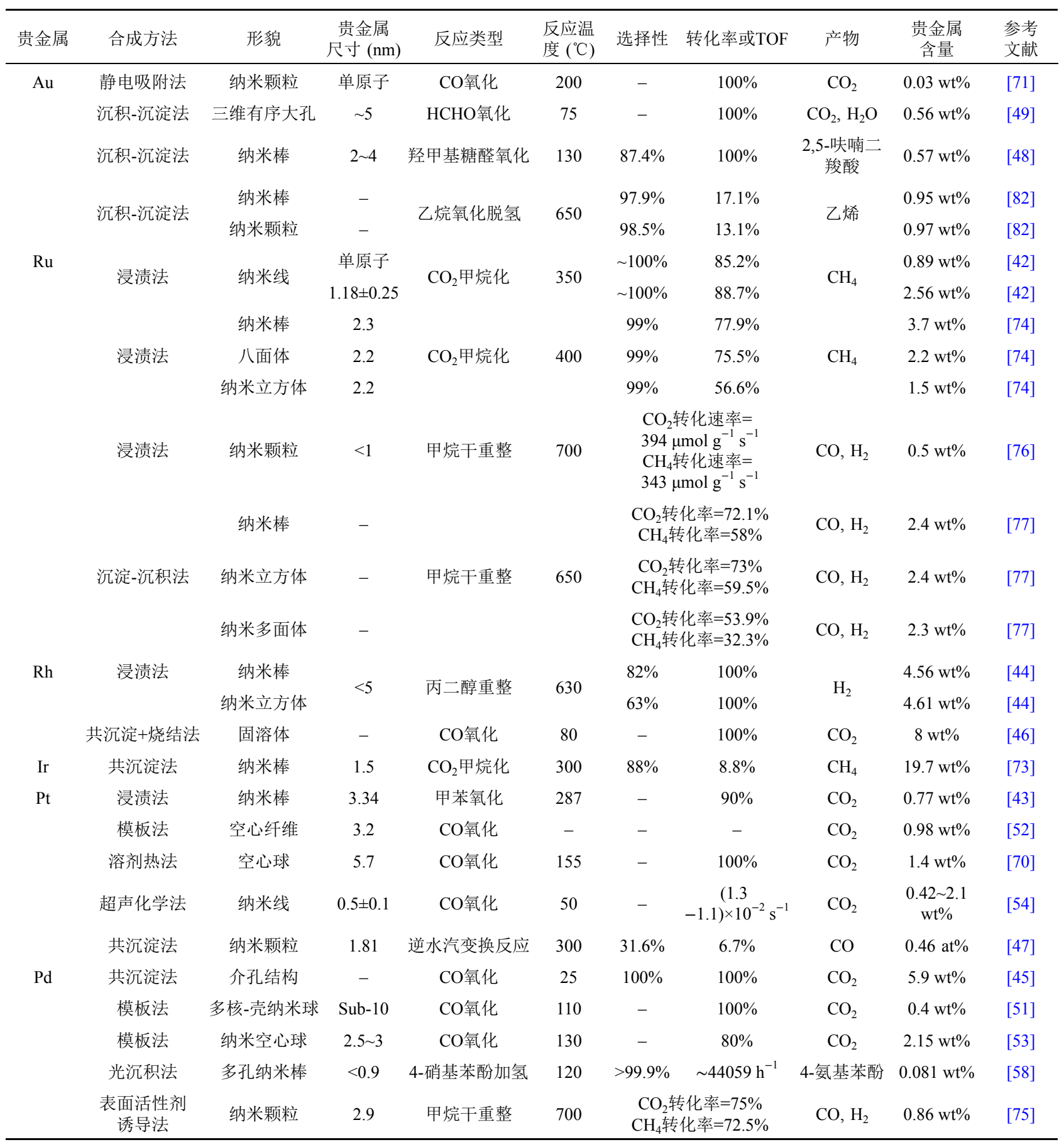

原子的浓度降低，阻碍了表面 $\mathrm{H}$ 原子和 $\mathrm{OH}$ 基团结合去 除水分子．这两种因素在 $\mathrm{CeO}_{2}$ 负载Ru纳米团簇(粒径 约为 $1.2 \mathrm{~nm}$ )催化剂中达到平衡，因此其 $\mathrm{CO}_{2}$ 甲烷化性
能最好. 类似地, $\mathrm{Li}$ 等 $^{\left[{ }^{[73]}\right.}$ 研究了 $\mathrm{Ir} / \mathrm{CeO}_{2}$ 催化剂中SMSI 效应对其 $\mathrm{CO}_{2}$ 选择性加氢性能的影响．通过改变金属 的负载量, 可以实现Ir化学状态的调控. 在 $\mathrm{CO}_{2}$ 加氢反 

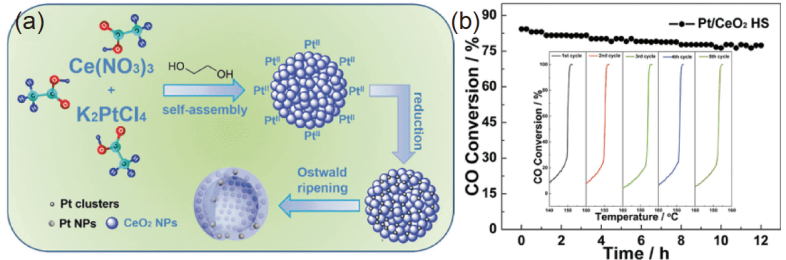

(c)
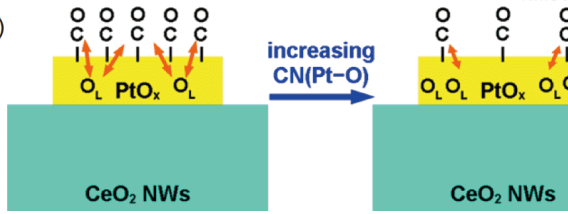

$O_{L} O_{L} P^{\prime} O_{x} O_{L} O_{L}$

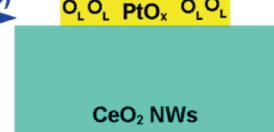

图 $13 \mathrm{Pt} / \mathrm{CeO}_{2} \quad \mathrm{HS}$ 纳米催化剂的合成示意图(a)和稳定性测 试曲线(b) ${ }^{[70]}$; (c) 亚纳米 $\mathrm{PtO}_{x} / \mathrm{CeO}_{2} \mathrm{NWs}$ 中 $\mathrm{CN}(\mathrm{Pt}-\mathrm{O})$ 增加对 $\mathrm{CO}$ 氧化的影响 ${ }^{[54]}$ (网络版彩图)

Figure 13 (a) Scheme of the synthetic procedure and (b) long-term stability test of $\mathrm{Pt} / \mathrm{CeO}_{2}$ HS nanocatalysts [70]; (c) the effects of the increasing $\mathrm{CN}(\mathrm{Pt}-\mathrm{O})$ of subnano $\mathrm{PtO}_{x} / \mathrm{CeO}_{2} \mathrm{NWs}$ on $\mathrm{CO}$ oxidation [54] (color online).

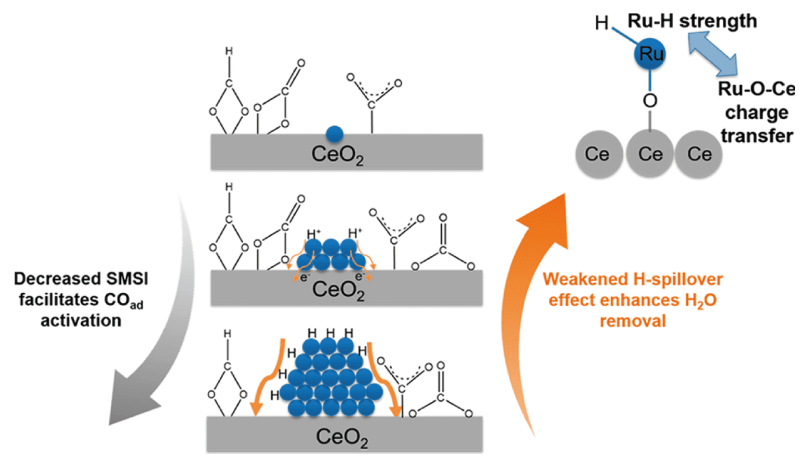

图 14 强金属-载体相互作用以及 $\mathrm{H}$ 溢流效应对 $\mathrm{Ru} / \mathrm{CeO}_{2}$ 负 载型催化剂催化 $\mathrm{CO}_{2}$ 甲烷化反应的影响示意图 ${ }^{[42]}$ (网络版 彩图)

Figure 14 Schematic illustration of the influences of SMSI and $\mathrm{H}-$ spillover effect towards the $\mathrm{CO}_{2}$ methanation reaction on $\mathrm{Ru} / \mathrm{CeO}_{2}$ catalysts [42] (color online).

应中，SMSI诱导的Ir物种的化学状态对反应的选择性 有很大的影响.

此外, $\mathrm{CeO}_{2}$ 载体的形貌也会影响贵金属-氧化铈负 载型催化剂在 $\mathrm{CO}_{2}$ 甲烷化中的性能. Sakpal等 ${ }^{[74]}$ 在 $\mathrm{CeO}_{2}$ 纳米棒 $(\mathrm{NR})$ 、立方体 $(\mathrm{NO})$ 和八面体上负载了特 定颗粒尺寸的 $\mathrm{Ru}$ ，并将这些催化剂焙烧后的氧空位浓 度的变化趋势与 $\mathrm{CO}_{2}$ 甲烷化反应活性趋势作对比. 研 究结果表明, 催化剂中氧空位浓度的顺序是 $\mathrm{Ru} / \mathrm{CeO}_{2}$ $\mathrm{NC}<\mathrm{Ru} / \mathrm{CeO}_{2}-\mathrm{NO}<\mathrm{Ru} / \mathrm{CeO}_{2}-\mathrm{NR}$, 这与 $\mathrm{CO}_{2}$ 转化顺序是 一致的, 进一步说明 $\mathrm{CO}_{2}$ 在氧空位处的活性吸附是反
应决速步．此外，氧缺陷和界面位点都会影响甲烷化 的性能, 对于 $\mathrm{Ru}$ 催化剂, 界面位点浓度为 $\mathrm{Ru} / \mathrm{CeO}_{2}-$ $\mathrm{NO}<\mathrm{Ru} / \mathrm{CeO}_{2}-\mathrm{NC}<\mathrm{Ru} / \mathrm{CeO}_{2}-\mathrm{NR} ，$ 这个顺序与甲烷化反 应的选择性趋势一致.

\subsection{3 甲烷干重整(DRM)}

碳氢键解离后, $\mathrm{CO}_{2}$ 与甲烷反应生成 $\mathrm{H}_{2} / \mathrm{CO}$ 比约为 1 的合成气，可进一步应用于商用化学试剂的工业生 产，如氨、甲醇、烯烃和芳烃等. 但是，由于反应物的 高稳定性, 甲烷干重整一般需要 $550^{\circ} \mathrm{C}$ 以上的高温. 贵 金属-氧化铈负载型催化剂结构稳定且充分暴露贵金 属活性位点, 因而被广泛用作DRM催化剂.

例如，Singha等 ${ }^{[75]}$ 采用简单的表面活性剂诱导法 制备了 $\mathrm{Pd}-\mathrm{CeO}_{2}$ 纳米晶，在 $350^{\circ} \mathrm{C}$ 下用于甲烷的干重整 反应. 所得的催化剂具有良好的低温活性, 主要是由 于高分散的 $\mathrm{Pd}$ 与 $\mathrm{CeO}_{2}$ 载体之间的强金属-载体相互作 用, 以及 $\mathrm{CeO}_{2}\left(32.8 \%\right.$ 的 $\mathrm{Ce}^{3+}$ 浓度)的良好的氧化还原性 能. 该催化剂在甲烷干重整催化过程中具有良好的抗 焦性和抑制结焦失活的能力. 然而, 连续反应 $12 \mathrm{~h}$ 测试 其稳定性，发现Pd纳米颗粒由于烧结、再氧化和羟基 化作用而使转化率略有下降.

同样地, 改变金属的颗粒尺寸, 在SMSI作用下, 会 改变催化剂的电子结构, 从而影响其甲烷干重整性能. 例如， $\mathrm{Liu}$ 等 ${ }^{[76]}$ 通过浸渍法在 $\mathrm{CeO}_{2}$ 表面负载了颗粒尺 寸 $<1 \mathrm{~nm}$ 的 $\mathrm{Ru}$ 纳米团簇 $(\mathrm{NCs})$ 和粒径为 $3 \sim 4 \mathrm{~nm}$ 的 $\mathrm{Ru}$ 纳 米颗粒(NP). $\mathrm{Ru}(\mathrm{NC})-\mathrm{CeO}_{2}$ 催化剂对甲烷的干重整表 现出优异的反应活性和稳定性，如图 15 所示. 与 $\mathrm{Ru}(\mathrm{NP})-\mathrm{CeO}_{2}$ 体系中金属性的 $\mathrm{Ru}$ NPs 不同, $\mathrm{Ru}(\mathrm{NC})-$ $\mathrm{CeO}_{2}$ 催化剂的反应活性结构是被还原性 $\mathrm{CeO}_{2}$ 稳定的 带部分电荷的 Ru NCs $\left(\mathrm{Ru}^{\delta+}-\mathrm{CeO}_{2-x}\right)$. 此外, 表面碳的 氧化是催化反应的关键步骤, 这与高温下 $\mathrm{Ru}^{\delta+}-\mathrm{CeO}_{2-x}$ 相互作用主导的氧转移有关.

氧化铈载体的形貌和暴露晶面也会对贵金属-氧 化铈负载型催化剂的甲烷干重整性能产生重要影响. $\mathrm{He}$ 等 ${ }^{[77]}$ 在 $\mathrm{CeO}_{2}$ 纳米棒 $(\mathrm{NRs})$ 、纳米立方体 $(\mathrm{NCs})$ 和纳 米多面体 $(\mathrm{NPs})$ 表面负载了 $\mathrm{Ru}$ 纳米颗粒. 实验结果表 明, $\mathrm{CeO}_{2}-\mathrm{NRs} 、 \mathrm{CeO}_{2}-\mathrm{NCs}$ 和 $\mathrm{CeO}_{2}-\mathrm{NPs}$ 分别主要暴露 (110)、(100)和(111)面, $\mathrm{CeO}_{2}-\mathrm{NRs}$ 和 $\mathrm{CeO}_{2}-\mathrm{NCs}$ 的氧空 位浓度高于 $\mathrm{CeO}_{2}-\mathrm{NPs}$. 在甲烷干重整中, $\mathrm{Ru} / \mathrm{CeO}_{2}-\mathrm{NR}$ 和 $\mathrm{Ru} / \mathrm{CeO}_{2}-\mathrm{NC}$ 催化剂的性能优于 $\mathrm{Ru} / \mathrm{CeO}_{2}-\mathrm{NPs}$, 说明 高氧空位浓度的催化剂有利于促进活性的提高. 

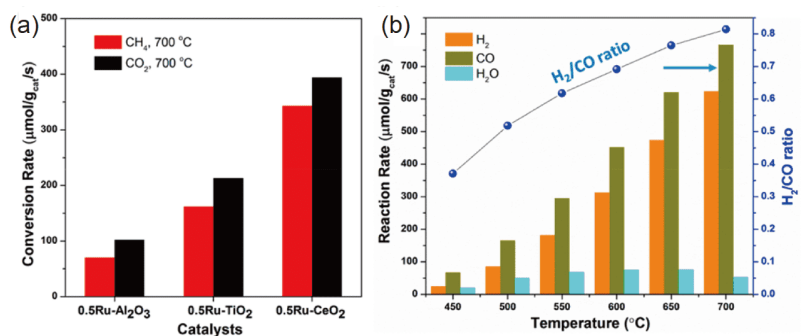

图 $15 \mathrm{Ru}(\mathrm{NC})-\mathrm{CeO}_{2}$ 催化剂的甲烷干重整性能 ${ }^{[76]}$ (网络版彩 图)

Figure 15 Catalytic performance of $\mathrm{Ru}(\mathrm{NC})-\mathrm{CeO}_{2}$ for dry reforming of methane [76] (color online).

\subsection{4 水汽变换(WGS) 反应}

水汽变换(WGS)反应被广泛用于从化石碳质和可 再生生物质原料中生产氢、从合成气中去除 $\mathrm{CO}$ 以净 化氢气，同时也是整个甲烷重整过程的一个组成部 分 ${ }^{[78]}$. WGS 反应式为: $\mathrm{CO}+\mathrm{H}_{2} \mathrm{O} \rightarrow \mathrm{H}_{2}+\mathrm{CO}_{2}\left(\Delta H^{\Theta}{ }_{298 \mathrm{~K}}=\right.$ $-41.09 \mathrm{~kJ} / \mathrm{mol})$. CO 和水在合适的催化剂上进行反应, 以使气态混合物中含有 $\mathrm{H}_{2}$. 金属和金属氧化物均可用 于催化WGS反应，贵金属-氧化铈负载型催化剂能够 发挥两者的协同作用, 因而成为常用的WGS催化剂.

如前所述, 强金属-载体相互作用和表面氧空位对 WGS反应性能也具有显著影响. 例如, Flytzani-Stephanopoulos课题组 ${ }^{[79]}$ 报道了 $\mathrm{Pt} / \mathrm{CeO}_{2}$ 和 $\mathrm{Au} / \mathrm{CeO}_{2}$ 纳米催化 剂用于低温WGSR. 研究结果表明, $\mathrm{Au}$ 物种通过 $\mathrm{CeO}_{2}$ 的表面缺陷被针定, $\mathrm{Au}$ 的加入增强了 $\mathrm{CeO}_{2}$ 载体表面氧 的可还原性, 表面可被还原的氧的数目与 $\mathrm{CeO}_{2}$ 的尺寸 有关. 特别地, 非金属性的 $\mathrm{Au}$ 和 $\mathrm{Pt}$ 物种与 $\mathrm{CeO}_{2}$ 表面的 $\mathrm{Ce}-\mathrm{O}$ 基团之间存在较强的相互作用, 这是促进WGS 反 应进行的主要因素. Cavusoglu等 ${ }^{[80]}$ 采用喷雾燃烧热解 法制备了 $\mathrm{CeO}_{2}$ 负载的 $\mathrm{Rh}$ 和Pt催化剂, 并测试了它们的 WGS 催化性能. 在所有情况下, $\mathrm{Pt} / \mathrm{CeO}_{2}$ 相较于 $\mathrm{Rh} / \mathrm{CeO}_{2}$ 催化剂均表现出较高的催化活性和选择性. 氧化铈载体的形貌和暴露晶面也会对贵金属-氧化铈 负载型催化剂的WGS性能产生重要影响. Cifuentes 等 ${ }^{[81]}$ 在具有特定形貌的 $\mathrm{CeO}_{2}$ (如多面体、纳米棒、纳 米立方体)和形貌不规则的 $\mathrm{CeO}_{2}$ 载体上分别负载了 $\mathrm{Au}-\mathrm{Cu}$ 得到 $\mathrm{AuCu} / \mathrm{CeO}_{2}$ 催化剂, 并将其用于合成气中的 $\mathrm{CO}$ 去除. $\mathrm{CeO}_{2}$ 纳米多面体和纳米棒具有较高的表面积 和 $\{111\} /\{100\}$ 晶面, 因此具有较高的活性和选择性. 其中, 只有 $\mathrm{AuCu} / \mathrm{CeO}_{2}$ 纳米多面体催化剂可以在 $48 \mathrm{~h}$ 内完全去除合成气中的CO $(<100 \mathrm{ppm})$. 这主要是由
于 $\mathrm{AuCu} / \mathrm{CeO}_{2}$ 纳米棒存在不稳定的 $\{110\}$ 晶面, 在长时 间反应后容易失去对 $\mathrm{CO}_{2}$ 的选择性.

综上所述, 贵金属-氧化铈负载型催化剂因其独特 且易于调控的结构和性质而在催化领域具有非常广泛 的应用. 影响其催化性能的主要因素有: (1) 贵金属的 尺寸和种类; (2) 氧化铈载体的形貌、结构、氧化还原 特性、表面缺陷和暴露晶面; (3) 贵金属与氧化铈之间 的相互作用, 包括强金属-载体相互作用、物种溢流效 应等. 深刻理解这些因素的催化作用机制对于我们后 续设计和开发高效、绿色、低成本的催化剂具有重要 指导意义.

\section{4 总结与展望}

本文从贵金属基纳米材料出发，结合本课题组的 相关研究工作, 主要介绍了双金属/三金属纳米催化剂 和贵金属-氧化铈负载型催化剂的控制合成、性能调 控及其在催化应用中的研究进展. 贵金属基纳米材料 由于其结构可调性和优异的物理化学性质在热催化和 电催化反应中具有广阔的应用前景.

双金属/三金属纳米催化剂相较于单组分贵金属 催化剂具有以下优点: (1) 催化剂组分可大范围调控; (2) 可以通过形貌调控、晶相调控、电子结构的调控 等多方面来改善催化剂性能; (3) 可减少贵金属使用 量, 降低催化剂成本. 随着现代分析仪器和原位表征 技术的发展，基于原子层面设计的催化剂也越来越受 到科研工作者的重视. 例如, 单原子合金催化剂成为 新的研究热点之一, 但是其尚处于初步研究阶段. 对 于单原子合金的精确可控制备以及结合原位表征测量 技术和DFT计算模拟深入理解其生长机理以及催化反 应机理还有待于进一步深入研究，从而制备出更高活 性、低成本的合金催化剂.

贵金属-氧化铈负载型催化剂能够在强金属-载体 相互作用下发挥贵金属与氧化铈之间的协同作用，在 催化领域具有巨大的应用潜力. 该复合催化剂能有效 降低贵金属使用量，且氧化铈在地壳中储量丰富，从 而使得其生产成本较低，通过进一步的升级开发，有 望在工业生产中大规模得到使用. 但是, 尺寸效应是 影响负载型催化剂催化活性的重要因素, 因此, 如何 实现不同尺寸催化剂(单原子、团簇、纳米颗粒)的精 确可控合成也是我们需要解决的问题之一. 同时, 贵金 
属/氧化铈负载型催化剂在绿色合成方法开发、反应 活性位点的确定、催化作用机理的认识等方面仍有待 于进一步深入研究. 此外, 将贵金属(单原子)合金与负 载型催化剂相结合以获得新型高效催化剂也是一个有
意义的研究趋势. 借助于DFT理论计算与原位表征技 术，深入挖掘其独特催化性质和反应机理，拓展其在 催化领域的应用, 将有力推动贵金属/氧化铈纳米材料 研究领域的快速发展.

\section{参考文献}

1 Yin AX, Liu WC, Ke J, Zhu W, Gu J, Zhang YW, Yan CH. J Am Chem Soc, 2012, 134: 20479-20489

2 Li WZ, Liu JX, Gu J, Zhou W, Yao SY, Si R, Guo Y, Su HY, Yan CH, Li WX, Zhang YW, Ma D. J Am Chem Soc, 2017, 139: 2267-2276

3 Jung N, Chung DY, Ryu J, Yoo SJ, Sung YE. Nano Today, 2014, 9: 433-456

4 Yang X, Skrabalak SE, Li ZY, Xia Y, Wang LV. Nano Lett, 2007, 7: 3798-3802

5 Taton TA, Mirkin CA, Letsinger RL. Science, 2000, 289: 1757-1760

6 Zhang YW. Bimetallic Nanostructures (Shape-Controlled Synthesis for Catalysis, Plasmonics, and Sensing Applications). New Jersey: John Wiley \& Sons Ltd, 2018

7 Gatalo M, Ruiz-Zepeda F, Hodnik N, Dražić G, Bele M, Gaberšček M. Nano Energy, 2019, 63: 103892

8 Cesar LG, Yang C, Lu Z, Ren Y, Zhang G, Miller JT. ACS Catal, 2019, 9: 5231-5244

9 Dai LX, Zhu W, Lin M, Zhang ZP, Gu J, Wang YH, Zhang YW. Inorg Chem Front, 2015, 2: 949-956

10 Rizo R, Sebastián D, Lázaro MJ, Pastor E. Appl Catal B-Environ, 2017, 200: 246-254

11 Li M, Cullen DA, Sasaki K, Marinkovic NS, More K, Adzic RR. J Am Chem Soc, 2013, 135: 132-141 11280

Michel J, Schwartz J. Stud Surf Sci Catal, 1987, 31: 669-687 
Jana NR, Gearheart L, Murphy CJ. Chem Mater, 2001, 13: 2313-2322

Yang M, Hood ZD, Yang X, Chi M, Xia Y. Chem Commun, 2017, 53: 1965-1968

Gu J, Guo Y, Jiang YY, Zhu W, Xu YS, Zhao ZQ, Liu JX, Li WX, Jin CH, Yan CH, Zhang YW. J Phys Chem C, 2015, 119: 17697-17706

Zhou JH, Lan DW, Yang SS, Guo Y, Yuan K, Dai LX, Zhang YW. Inorg Chem Front, 2018, 5: 1524-1532

Yin AX, Min XQ, Zhu W, Liu WC, Zhang YW, Yan CH. Chem Eur J, 2012, 18: 777-782

Guo Y, Mei S, Yuan K, Wang DJ, Liu HC, Yan CH, Zhang YW. ACS Catal, 2018, 8: 6203-6215

Wang B, Chen B, Sun Y, Xiao H, Xu X, Fu M, Wu J, Chen L, Ye D. Appl Catal B-Environ, 2018, 238: 328-338

Shanmugam V, Zapf R, Hessel V, Pennemann H, Kolb G. Appl Catal B-Environ, 2018, 226: 403-411

Li G, Li L, Jiang D, Li Y, Shi J. Nanoscale, 2015, 7: 5691-5698

Derevyannikova EA, Kardash TY, Kibis LS, Slavinskaya EM, Svetlichnyi VA, Stonkus OA, Ivanova AS, Boronin AI. Phys Chem Chem Phys, 2017, 19: 31883-31897

Chen X, Su X, Liang B, Yang X, Ren X, Duan H, Huang Y, Zhang T. J Energy Chem, 2016, 25: 1051-1057

Li Q, Wang H, Tian Z, Weng Y, Wang C, Ma J, Zhu C, Li W, Liu Q, Ma L. Catal Sci Technol, 2019, 9: 1570-1580

Liu B, Li C, Zhang Y, Liu Y, Hu W, Wang Q, Han L, Zhang J. Appl Catal B-Environ, 2012, 111-112: 467-475

Tinoco M, Fernandez-Garcia S, Lopez-Haro M, Hungria AB, Chen X, Blanco G, Perez-Omil JA, Collins SE, Okuno H, Calvino JJ. ACS Catal, 2015, 5: 3504-3513

Chen C, Fang X, Wu B, Huang L, Zheng N. ChemCatChem, 2012, 4: 1578-1586

Yoon K, Yang Y, Lu P, Wan D, Peng HC, Stamm Masias K, Fanson PT, Campbell CT, Xia Y. Angew Chem Int Ed, 2012, 51: 9543-9546

Du C, Guo Y, Guo Y, Gong X, Lu G. J Mater Chem A, 2015, 3: 23230-23239

Ke J, Zhu W, Jiang Y, Si R, Wang YJ, Li SC, Jin C, Liu H, Song WG, Yan CH, Zhang YW. ACS Catal, 2015, 5: 5164-5173

Shukla A, Singha RK, Sasaki T, Bal R. Green Chem, 2015, 17: 785-790

Liu J, Wang W, Shen T, Zhao Z, Feng H, Cui F. RSC Adv, 2014, 4: 30624-30629

Simon P, Zanfoni N, Avril L, Li Z, Potin V, Domenichini B, Bourgeois S. Adv Mater Interfaces, 2017, 4: 1600821

Zhang S, Chang CR, Huang ZQ, Li J, Wu Z, Ma Y, Zhang Z, Wang Y, Qu Y. J Am Chem Soc, 2016, 138: 2629-2637

Gu J, Zhang YW, Tao FF. Chem Soc Rev, 2012, 41: 8050-8065

Yin HJ, Zhou JH, Zhang YW. Inorg Chem Front, 2019, 6: 2582-2618

Yin AX, Min XQ, Zhang YW, Yan CH. J Am Chem Soc, 2011, 133: 3816-3819

Yin AX, Min XQ, Zhu W, Wu HS, Zhang YW, Yan CH. Chem Commun, 2012, 48: 543-545

Dai LX, Wang XY, Zheng XY, Zhang YW. Chem Commun, 2016, 52: 5023-5026

Gu J, Liu WC, Zhao ZQ, Lan GX, Zhu W, Zhang YW. Inorg Chem Front, 2014, 1: 109-117

Erini N, Beermann V, Gocyla M, Gliech M, Heggen M, Dunin-Borkowski RE, Strasser P. Angew Chem Int Ed, 2017, 56: 6533-6538

Gloag L, Benedetti TM, Cheong S, Marjo CE, Gooding JJ, Tilley RD. J Am Chem Soc, 2018, 140: 12760-12764

Feng J, Lv F, Zhang W, Li P, Wang K, Yang C, Wang B, Yang Y, Zhou J, Lin F, Wang GC, Guo S. Adv Mater, 2017, 29: 1703798

Shan J, Zhang S, Choksi T, Nguyen L, Bonifacio CS, Li Y, Zhu W, Tang Y, Zhang Y, Yang JC, Greeley J, Frenkel AI, Tao F. ACS Catal, 2017, 7: $191-204$

69 Zhu W, Shan J, Nguyen L, Zhang S, Tao FF, Zhang YW. Sci China Mater, 2019, 62: 103-114

Wu K, Zhou L, Jia CJ, Sun LD, Yan CH. Mater Chem Front, 2017, 1: 1754-1763

Zhao S, Chen F, Duan S, Shao B, Li T, Tang H, Lin Q, Zhang J, Li L, Huang J, Bion N, Liu W, Sun H, Wang AQ, Haruta M, Qiao B, Li J, Liu J, Zhang T. Nat Commun, 2019, 10: 3824

Parastaev A, Muravev V, Huertas Osta E, van Hoof AJF, Kimpel TF, Kosinov N, Hensen EJM. Nat Catal, 2020, 3: 526-533

Li S, Xu Y, Chen Y, Li W, Lin L, Li M, Deng Y, Wang X, Ge B, Yang C, Yao S, Xie J, Li Y, Liu X, Ma D. Angew Chem Int Ed, 2017, 56: $10761-10765$

Sakpal T, Lefferts L. J Catal, 2018, 367: 171-180

Singha RK, Yadav A, Shukla A, Kumar M, Bal R. Catal Commun, 2017, 92: 19-22

Liu Z, Zhang F, Rui N, Li X, Lin L, Betancourt LE, Su D, Xu W, Cen J, Attenkofer K, Idriss H, Rodriguez JA, Senanayake SD. ACS Catal, 2019, 9: 3349-3359 
Fu Q, Saltsburg H, Flytzani-Stephanopoulos M. Science, 2003, 301: 935-938

Cavusoglu G, Miao D, Lichtenberg H, Carvalho HWP, Xu H, Goldbach A, Grunwaldt JD. Appl Catal A-Gen, 2015, 504: 381-390

Cifuentes B, Bustamante F, Araiza DG, Diaz G, Cobo M. Appl Catal A-Gen, 2020, 598: 117568

Lei T, Miao C, Hua W, Yue Y, Gao Z. Catal Lett, 2018, 148: 1634-1642

\title{
Research progress on high-performance noble metal/cerium oxide nanocatalysts: shape controlled synthesis, performance regulation and applications
}

\author{
Qian Zhang, Yawen Zhang*
}

Beijing National Laboratory for Molecular Sciences, State Key Laboratory of Rare Earth Materials Chemistry and Applications, College of Chemistry and Molecular Engineering, Peking University, Beijing 100871, China.

*Corresponding author (email: ywzhang@pku.edu.cn)

\begin{abstract}
Noble metals are widely used in many fields, particularly in catalysis, due to their unique physical and chemical properties. However, their large-scale applications are limited, due to the high price, low reserves, easy agglomeration and the sintering of single component. The above problems can be effectively solved by constructing metal alloy nanocrystals and cerium oxide supported noble metal catalysts. In this review, we will summarize recent progress on the controlled synthesis and catalytic performances of noble metals/cerium oxide nanocatalysts. In detail, mainly based on our group's work, we present the controlled synthetic approaches, the relationship between the structures and properties, and the catalytic applications of those catalysts with specific shapes and nanostructures. Finally, the future developments and challenges of the cutting-edge research on noble metal-based catalysts are prospected.
\end{abstract}

Keywords: noble metal, cerium oxide, controllable synthesis, catalysis

doi: $10.1360 /$ SSC-2020-0135 